Alain-Sol Sznitman

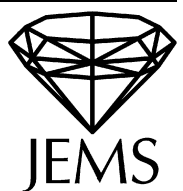

\title{
Slowdown estimates and central limit theorem for random walks in random environment
}

Received May 31, 1999 / final version received January 18, 2000

Published online April 19, 2000 - (c) Springer-Verlag \& EMS 2000

\begin{abstract}
This work is concerned with asymptotic properties of multi-dimensional random walks in random environment. Under Kalikow's condition, we show a central limit theorem for random walks in random environment on $\mathbb{Z}^{d}$, when $d \geq 2$. We also derive tail estimates on the probability of slowdowns. These latter estimates are of special interest due to the natural interplay between slowdowns and the presence of traps in the medium. The tail behavior of the renewal time constructed in [25] plays an important role in the investigation of both problems. This article also improves the previous work of the author [24], concerning estimates of probabilities of slowdowns for walks which are neutral or biased to the right.
\end{abstract}

\section{Introduction}

Random walk in a $d$-dimensional random environment is a basic example of stochastic motion in a random medium. Yet, its asymptotic behavior is so far rather poorly understood, especially when $d$ is bigger than one. The concept of the "environment viewed from the particle", which has been so powerful in the investigation of several examples of stochastic motions in a random environment, cf. Kipnis-Varadhan [13], S.M. Kozlov [14], Olla [18], Papanicolaou-Varadhan [19], has had until now little success in the study of multidimensional random walks in random environment. This fact is related to the genuinely non-reversible character of the model, and to the difficulty to apply any ergodic theorem.

In Sznitman and Zerner [25], it is shown that an assumption previously introduced by Kalikow [9], see also (1.7) below, implies a strong law of large numbers for the walk, with a non-degenerate limiting velocity. The object of the present article is to investigate under the above assumption, the tail behavior of probabilities of slowdown of the walk, and conditions ensuring a central limit theorem. The wish to derive a central limit theorem is very natural and there is little need to further dwell on its motivation. Let us however comment on the incentive to study the tail behavior of 
the probability of slowdowns, that is to obtain large deviation estimates on the probability that the walk moves slower than predicted by the law of large numbers. This topic has recently been the object of several works mainly in a one-dimensional setting, cf. Dembo-Peres-Zeitouni [4], GantertZeitouni [7], Pisztora-Povel-Zeitouni [21], Pisztora-Povel [20], with the exception of Sznitman [24], where a multi-dimensional situation is analyzed. One ground for this surge of interest is the profound interplay between probabilities of slowdown of the walk, and the nature of "traps" which can occur in the medium. Loosely speaking the traps are atypical pockets of the medium where the walk may spend a long time with relatively large probability. The desire to elucidate the role of such objects in the tail behavior of probability of slowdowns can be viewed as part of a broader issue in the theory of random media. Indeed over the recent years, several important examples have emerged, where "atypical pockets of low local eigenvalues" play a predominant role. Such examples can for instance be found in the context of stochastic dynamics of spin systems with random interaction, cf. Sect. 7 of Martinelli [16], of models of intermittency, cf. Chap. III of Molchanov [17], and of Brownian motion among Poissonian obstacles, cf. Sznitman [23]. One of the aspirations of the present work is to study whether traps play a predominent role in slowdowns.

Let us now present the model more precisely. The random environment is given by a collection of i.i.d. (2d)-dimensional vectors which specify the jump probabilities of the walk at each site of $\mathbb{Z}^{d}$. We assume that for a suitable $\kappa$ in $\left(0, \frac{1}{2 d}\right]$,

the common law $\mu$ of the vectors is supported on the set $\mathcal{P}_{\kappa}$

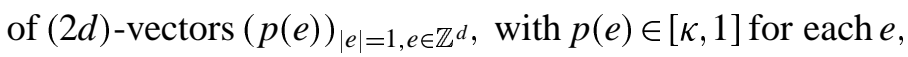
and $\sum_{|e|=1} p(e)=1$.

The random environment is an element $\omega=(\omega(x, \cdot))_{x \in \mathbb{Z}^{d}}$ of $\Omega=\mathcal{P}_{\kappa}^{\mathbb{Z}^{d}}$, which is tacitly endowed with the product $\sigma$-algebra and the product measure $\mathbb{P}=\mu^{\otimes \mathbb{Z}^{d}}$. The random walk in the random environment $\omega$, is then the canonical Markov chain $\left(X_{n}\right)_{n \geq 0}$ on $\left(\mathbb{Z}^{d}\right)^{\mathbb{N}}$, with state space $\mathbb{Z}^{d}$, and "quenched law" $P_{x, \omega}$ starting from $x \in \mathbb{Z}^{d}$, such that:

$$
\begin{aligned}
& P_{x, \omega}\left[X_{n+1}=X_{n}+e \mid X_{0}, \ldots, X_{n}\right] \stackrel{P_{x, \omega} \text {-a.s. }}{=} \omega\left(X_{n}, e\right), \\
& \text { for } n \geq 0, \text { and }|e|=1, P_{x, \omega}\left[X_{0}=x\right]=1 .
\end{aligned}
$$

One also defines the "annealed laws" $P_{x}, x \in \mathbb{Z}^{d}$, on $\Omega \times\left(\mathbb{Z}^{d}\right)^{\mathbb{N}}$ via

$$
P_{x}=\mathbb{P} \times P_{x, \omega}, x \in \mathbb{Z}^{d} .
$$


Throughout this article, the walks we consider will always fulfill Kalikow's condition relative to some unit vector $\ell$ of $\mathbb{R}^{d}$. We refer to (1.7) for the precise definition. As mentioned above, it is shown in Sznitman-Zerner [25], that this condition implies a strong law of large number, namely:

$$
P_{0} \text {-a.s, } \frac{X_{n}}{n} \rightarrow v,
$$

where the limiting velocity $v$, cf. (1.21), is deterministic and $v \cdot \ell>0$. To highlight the nature of Kalikow's condition, let us mention that in the onedimensional situation, under (0.1), a strong law of large numbers always holds for random walks in random environment, but the limit velocity possibly vanishes, cf. Solomon [22]. The fulfillment of Kalikow's condition with respect to $\ell=1$, or $\ell=-1$, precisely characterizes the case of a nonvanishing limit velocity, cf. Remark 2.5 of [25]. Unfortunately the higher dimensional situation is less clear, so far.

Coming back to the main objectives of the present article, let us define for $u \in \mathbb{R}$ :

$$
T_{u}=\inf \left\{n \geq 0, X_{n} \cdot \ell \geq u\right\},
$$

the first time at which the walk comes above level $u$ in the direction $\ell$. The strong law of large numbers is easily seen to imply that:

$$
P_{0} \text {-a.s., } \frac{T_{u}}{u} \rightarrow(v \cdot \ell)^{-1}, \text { as } u \rightarrow \infty .
$$

The central object of the present work is to investigate the asymptotics of the largely deviant annealed probabilities of slowdown:

$$
P_{0}\left[T_{u}>c u\right] \text {, as } u \rightarrow \infty, \text { with } c>(v \cdot \ell)^{-1},
$$

as well as the occurrence of a functional central limit theorem for:

$$
B_{t}^{n}=\frac{X_{[t n]}-[t n] v}{\sqrt{n}}, t \geq 0 \text {, when } n \rightarrow \infty .
$$

A powerful tool to analyze these questions is provided by the renewal structure constructed in [25], which generalizes to a multi-dimensional context the work of Kesten [11]. In particular a key role is played by a certain renewal time $\tau_{1}$, see (1.18) below. Both asymptotics in (0.7) and (0.8) are efficiently controlled by the tail behavior of $\tau_{1}$ :

$$
P_{0}\left[\tau_{1}>u\right], \text { as } u \rightarrow \infty .
$$

For instance, a finite second moment of $\tau_{1}$ implies a functional central limit theorem for $B^{n}$, cf. Theorem 4.1, and certain bounds on the tail of $\tau_{1}$ imply analogous bounds on the quantity in (0.7), cf. Lemma 5.1, Theorem 5.3 and Theorem 5.7. It follows from Theorem 3.5, that $\tau_{1}$ has finite $P_{0}$-moments of 
arbitrary order, when $d \geq 2$, and the central limit theorem for $B^{n}$ follows, cf. Corollary 4.2. This is in contrast with the one-dimensional situation where such a central limit theorem need not always hold, cf. Kesten-KozlovSpitzer [12]. Incidentally a central limit theorem for multi-dimensional random walks in random environment was already derived by Lawler [15], see also S.M. Kozlov [14], when the walk has null local drift, cf. (0.11), and by Bricmont-Kupiainen [2], when $d \geq 3$, the law $\mu$ is isotropic, (i.e. invariant under rotations preserving $\mathbb{Z}^{d}$ ), and concentrated on small perturbations of the transition probability of the simple random walk. The situation we consider here is quite different since an effective velocity exists.

The quantity in (0.9) has some similarities with $S(u)$, the annealed survival probability up to time $u$, which one considers in the context of Brownian motion among Poissonian obstacles, cf. Sznitman [23], Chap. 4. This qualitative analogy can be sensed once one realizes that a natural lower bound on (0.9) is obtained by writing

$$
P_{0}\left[\tau_{1}>u\right] \geq P_{0}\left[T_{U}>u, X_{u}=0\right],
$$

for $u$ an even integer (for parity reasons), $U$ an arbitrary subset of $\mathbb{Z}^{d}$ containing $0, T_{U}$ the exit time of $\left(X_{n}\right)_{n \geq 0}$ from $U$, and then picking a favorable, trap-like environment on $U$. This has of course very much the flavor of the type of lower bounds one applies to $S(u)$, when one constrains Brownian motion not to exit a large open set $U$ receiving no obstacles, up to time $u$, cf. [23].

In both cases upper bounds tend to be of a substantially more delicate nature than lower bounds. In the context of Brownian motion among Poissonian obstacles, upper bounds on $S(u)$ are very efficiently derived by means of certain large deviation estimates on principal Dirichlet eigenvalues, cf. [23]. However spectral consideration are much less quantitative in the truly non-self adjoint setting of random walks in random environment. Instead an important role in the derivation of upper bounds on (0.9), is played by certain large deviation estimates on the $\mathbb{P}$-probability that the exit distribution of a large slab $\left\{z \in \mathbb{Z}^{d},|z \cdot \ell| \leq L\right\}$, under $P_{0, \omega}$, gives "little mass" to the "upper boundary", where $z \cdot \ell>L$, cf. (2.15) and Proposition 3.1.

A fascinating feature of both tail behaviors in (0.7) and (0.9) is their profound link with the nature of possible traps which can occur in the medium. In particular if $d(x, \omega)$ denotes the local drift at $x$ in the environment $\omega$ :

$$
d(x, \omega)=E_{x, \omega}\left[X_{1}-X_{0}\right]=\sum_{|e|=1} \omega(x, e) e,
$$

a decisive role is played by

(0.12) $\quad K_{o}$ the convex hull of the support of the law of $d(0, \omega)$. 
Depending on whether the walk is

i) non-nestling, i.e. 0 does not belong to $K_{o}$ (the terminology is due to Zerner [26]),

ii) marginal nestling, i.e. 0 belongs to $\partial K_{o}$,

iii) plain nestling, i.e. 0 belongs to the interior of $K_{o}$,

one obtains both in (0.7) and (0.9) qualitatively distinct tail behaviors with respect to suitable directions $\ell$. Incidentally one interest of Kalikow's condition is that it is general enough to accommodate examples of the above three different classes. In case i) for directions $\ell$ separating 0 and $K_{o}$, the decay of $P_{0}\left[\tau_{1}>u\right]$ is exponential. In case iii), it is plausible to expect a typical behavior like $\exp \left\{-c(\log u)^{d}\right\}$. We can only show a lower bound of this type, cf. Theorem 2.7, and obtain when $d \geq 2$ an upper bound of the type $\exp \left\{-c(\log u)^{\alpha}\right\}$, with $\alpha$ smaller than $1+\frac{d-1}{3 d}$, cf. Theorem 3.5. In case ii), no universal decay should be expected, cf. Remark 2.6., although the situation is shown to be in an adequate sense intermediate between i) and iii). One important example of type ii) corresponds to walks which are neutral or biased to the right, cf. after (2.37), in which case $P_{0}\left[\tau_{1}>u\right]$ decays like $\exp \left\{-c u^{\frac{d}{d+2}}\right\}$, see Theorem 2.5. This example was in fact the main focus of our previous work [24]. Let us mention that the results we obtain in this special example improve our previous results and in particular enable to study tail behaviors like (0.7) for $c$ arbitrarily close to the critical value $(v \cdot \ell)^{-1}$, cf. Theorem 5.8. Parenthetically, the technique we employ here bypasses the renormalization method we used in [24].

Let us now turn to the organization of the present article.

Section I describes Kalikow's condition and the renewal structure which can be attached to the walk when the condition holds. It also develops certain estimates which are routinely used in the subsequent sections. We in particular show in Proposition 1.4 that $\sup _{0 \leq n \leq \tau_{1}}\left|X_{n \wedge \tau_{1}}\right|$ always has some finite exponential moment under $P_{0}$, (in contrast to $\tau_{1}$ in case (0.13) ii) and iii)). We also provide controls on the size of transversal fluctuations of the path, cf. Corollary 1.5 .

Section II discusses some first tail estimates on the variable $\tau_{1}$. Among other things it is shown that the three subcases of (0.13), lead for suitable directions $\ell$ to distinct tail behaviors. The special case of walks which are neutral or biased to the right is treated in a quite satisfactory fashion in Theorem 2.5.

Section III develops a priori upper bounds on the tail of $\tau_{1}$, when $d \geq 2$, with a special aim at the plain nestling situation. Our main result is provided 
in Theorem 3.5. Unfortunately the upper bounds we derive do not match the (natural) lowerbound we obtain in Theorem 2.7, (roughly following (0.10)).

Section IV applies the previously obtained tail estimates to the derivation of a central limit theorem for $B^{n}$, cf. Corollary 4.2.

Section V develops the applications of the tail estimates for (0.9), to the control of probabilities of slowdown. As mentioned above, we in particular drastically improve our previous results of [24], cf. Theorem 5.8. Our main focus in this section concerns annealed estimates. At the end of the section we briefly discuss some quenched estimates, and what happens in the case where one replaces "slowdown" by "acceleration", cf. Remark 5.9. For nestling walks satisfying Kalikow's condition, we also provide in Proposition 5.10 the description of the null set of the rate function entering the quenched large deviation principle of Zerner [26].

\section{Further notations and some preliminary estimates}

In this section we shall introduce some further notations, recall Kalikow's condition and the basic steps in the construction of the renewal structure introduced in [9]. We shall then prove several estimates which will be used in the subsequent sections. We begin with some notations. We respectively denote by $|\cdot|$ and $\|\cdot\|$ the Euclidean and the $\ell_{1}$-distance on $\mathbb{R}^{d},(d \geq 1)$, so that:

$$
|w| \leq\|w\| \leq \sqrt{d}|w|, \text { for } w \in \mathbb{R}^{d} .
$$

For $U$ a subset of $\mathbb{Z}^{d}$, we let $\partial U$ stand for the boundary of $U$ :

$$
\partial U=\left\{x \in \mathbb{Z}^{d} \backslash U, \exists y \in U,|y-x|=1\right\},
$$

and we denote by $T_{U}$ and $H_{U}$ the respective exit time and entrance time of $X$. in $U$ :

$$
T_{U}=\inf \left\{n \geq 0, X_{n} \notin U\right\}, H_{U}=\inf \left\{n \geq 0, X_{n} \in U\right\} .
$$

When $U=\{x\}$, we shall write $H_{x}$ instead of $H_{\{x\}}$, for simplicity. We now introduce a collection of auxiliary Markov chains which will be repeatedly used in the sequel, and also enter the definition of Kalikow's condition. For $U$ a connected strict subset of $\mathbb{Z}^{d}$, containing 0 , we consider the Markov chain with state space $U \cup \partial U$ and transition probability:

$$
\begin{aligned}
& \widehat{P}_{U}(x, x+e)= \\
& \mathbb{E}\left[E_{0, \omega}\left[\sum_{0}^{T_{U}} 1\left\{X_{n}=x\right\}\right] \omega(x, e)\right] / \mathbb{E}\left[E_{0, \omega}\left[\sum_{0}^{T_{U}} 1\left\{X_{n}=x\right\}\right]\right], \\
& \quad \text { for } x \in U,|e|=1, \widehat{P}_{U}(x, x)=1, x \in \partial U
\end{aligned}
$$


Thanks to (0.1) and the connectedness of $U$, the expectations entering (1.4) are readily seen to be finite and positive. The law of the canonical Markov chain attached to $\widehat{P}_{U}(\cdot, \cdot)$, and starting from $x \in U \cup \partial U$ is denoted by $\widehat{P}_{x, U}$. Its local drift at site $x \in U \cup \partial U$ is:

$$
\widehat{d}_{U}(x)=\widehat{E}_{x, U}\left[X_{1}-X_{0}\right] .
$$

The importance of these auxiliary Markov chains stems from the following fact proven in Proposition 1 of Kalikow [9]:

$$
\begin{aligned}
& \text { if } \widehat{P}_{0, U}\left[T_{U}<\infty\right]=1 \text {, then } P_{0}\left[T_{U}<\infty\right]=1 \text {, and } \\
& X_{T_{U}} \text { has same distribution under } \widehat{P}_{0, U} \text { and } P_{0} .
\end{aligned}
$$

For $\ell$ a unit vector of $\mathbb{R}^{d}$, Kalikow's condition relative to $\ell$ is the requirement that:

$$
\epsilon(\ell, \mu) \stackrel{\text { def }}{=} \inf _{U, x \in U} \widehat{d}_{U}(x) \cdot \ell>0,
$$

where $U$ runs over all possible connected strict subsets of $\mathbb{Z}^{d}$, containing 0 . Note that for a given $\mu$, the set of $\ell$ where (1.7) holds, is an open subset of $S^{d-1}$. Examples where (1.7) holds can be found in Sect. II, as well as in Kalikow [9] and Sznitman-Zerner [25]. Let us simply mention that (1.7) is general enough and can accommodate examples of distributions $\mu$ in the three different classes of (0.13).

From now on, we shall only consider situations where $\ell$ and $\mu$ fulfill (1.7). We denote by $C$ the closed convex cone

$$
C=\left\{w \in \mathbb{R}^{d},|w-w \cdot \ell \ell| \leq \frac{1}{\epsilon} w \cdot \ell\right\} .
$$

As a direct consequence of (1.7), we see that for any $U$ as in (1.7):

$$
\widehat{d}_{U}(x) \in C, \text { for } x \in U \cup \partial U \text {. }
$$

If $\left(\mathcal{F}_{n}\right)_{n \geq 0}$ stands for the canonical filtration of $\left(X_{n}\right)_{n \geq 0}$, a very useful role is played by the $\widehat{P}_{x, U}$-martingales, for $x \in U \cup \partial U$ :

$$
M_{n}^{U}=X_{n}-X_{0}-\sum_{0}^{n-1} \widehat{d}_{U}\left(X_{k}\right)
$$

These martingales have increments bounded in $|\cdot|$-norm by 2 , and from Azuma's inequality, cf. Alon-Spencer-Erdös [1], p. 85,

(1.11) $\widehat{P}_{x, U}\left[M_{n}^{U} \cdot w>A\right] \leq \exp \left\{-\frac{A^{2}}{8 n}\right\}$, for $A>0, n \geq 0,|w|=1$.

Observe also that $\sum_{0}^{n-1} \widehat{d}_{U}\left(X_{k}\right) \in C$, for all $n \geq 0, \widehat{P}_{x, U}$-a.s. . 
We now turn to the renewal structure attached to the random walk in random environment. It can in fact be constructed under more general assumptions than (1.7), we refer on this to [25]. If one introduces the stopping time

$$
D=\inf \left\{n \geq 0, \ell \cdot X_{n}<\ell \cdot X_{0}\right\},
$$

it can be shown, cf. Proposition 1.2 of [25] that:

$$
P_{0}[D=\infty]>0 .
$$

We shall in fact provide a quantitative lower bound on $P_{0}[D=\infty]$ in Lemma 1.1 below. The definition of the key random variable $\tau_{1}$ further depends on the choice of a number $a>0$. To get rid of the $a$ dependence in the estimates, it will be convenient and sufficient for our purpose to assume that

$$
0<a \leq 10 \sqrt{d},
$$

although nothing special happens when $a>10 \sqrt{d}$. We denote by $\left(\theta_{n}\right)_{n \geq 0}$, the canonical shift on $\left(\mathbb{Z}^{d}\right)^{\mathbb{N}}$ (or sometimes on $\left.(U \cup \partial U)^{\mathbb{N}}\right)$. Following Sect. I of [25], we introduce two sequences of $\left(\mathcal{F}_{n}\right)$-stopping times, $S_{k}, k \geq 0, R_{k}$, $k \geq 1$, and the sequence of successive maxima in the direction $\ell, M_{k}, k \geq 0$ :

$$
\begin{aligned}
& S_{0}=0, M_{0}=\ell \cdot X_{0}, \\
& S_{1}=T_{M_{0}+a} \leq \infty, R_{1}=D \circ \theta_{S_{1}}+S_{1} \leq \infty,
\end{aligned}
$$

(recall (0.5) for the notation),

$$
\begin{aligned}
& M_{1}=\sup \left\{\ell \cdot X_{n}, 0 \leq n \leq R_{1}\right\} \leq \infty, \\
& \quad \text { and by induction when } k \geq 1: \\
& S_{k+1}=T_{M_{k}+a} \leq \infty, R_{k+1}=D \circ \theta_{S_{k+1}}+S_{k+1} \leq \infty, \\
& M_{k+1}=\sup \left\{\ell \cdot X_{n}, 0 \leq n \leq R_{k+1}\right\} .
\end{aligned}
$$

We then have:

$$
0=S_{0} \leq S_{1} \leq R_{1} \leq S_{2} \leq \cdots \leq \infty,
$$

and the above inequalities are strict when the left member is finite. If we introduce

$$
K=\inf \left\{k \geq 1, S_{k}<\infty, R_{k}=\infty\right\},
$$

it is shown in Proposition 1.2. of [25] that:

$$
P_{0} \text {-a.s., } K<\infty \text {. }
$$


One then defines the positive variable (which is easily seen not to be an $\left(\mathcal{F}_{n}\right)$-stopping time):

$$
\tau_{1}=S_{K}
$$

Incidentally observe that the lower bound (0.10) immediately follows from the above definition. One then further introduces the successive times $\tau_{k}$, $k \geq 2$, (using hopefully obvious notations):

$$
\tau_{2}=\tau_{1}(X)+\tau_{1}\left(X_{\tau_{1}+\cdot}-X_{\tau_{1}}\right),
$$

( $\tau_{2}$ is defined as $+\infty$, when $\tau_{1}=\infty$ ), and for $k \geq 2$,

$$
\tau_{k+1}=\tau_{1}(X)+\tau_{k}\left(X_{\tau_{1}+\cdot}-X_{\tau_{1}}\right) .
$$

It is shown in Theorem 1.4 of [25] that

$$
P_{0} \text {-a.s., } 0<\tau_{1}<\tau_{2}<\cdots<\tau_{k}<\ldots \text {, and }
$$

under $P_{0},\left(\left(X_{\tau_{1} \wedge .}\right), \tau_{1}\right),\left(\left(X_{\left(\tau_{1}+\cdot\right) \wedge \tau_{2}}-X_{\tau_{1}}\right), \tau_{2}-\tau_{1}\right), \ldots$, $\left(\left(X_{\left(\tau_{k}+\cdot\right) \wedge \tau_{k+1}}-X_{\tau_{k}}\right), \tau_{k+1}-\tau_{k}\right), \ldots$, are independent

(1.19) variables, furthermore the $\left(\left(X_{\left(\tau_{1}+\cdot\right) \wedge \tau_{2}}-X_{\tau_{1}}\right), \tau_{2}-\tau_{1}\right), \ldots$, $\left(\left(X_{\left(\tau_{k}+\cdot\right) \wedge \tau_{k+1}}-X_{\tau_{k}}\right), \tau_{k+1}-\tau_{k}\right), \ldots$, are distributed like $\left(\left(X_{\tau_{1} \wedge}\right), \tau_{1}\right)$ under $P_{0}[\cdot \mid D=\infty]$.

The above renewal property will be sufficient for the purpose of the present article, but more is known, see [25]. It is then shown in Theorem 2.3 of [25], that:

$$
E_{0}\left[\tau_{1} \mid D=\infty\right]<\infty,
$$

as well as the strong law of large numbers:

$$
P_{0} \text {-a.s., } \frac{X_{n}}{n} \rightarrow v \stackrel{\text { def }}{=} \frac{E_{0}\left[X_{\tau_{1}} \mid D=\infty\right]}{E_{0}\left[\tau_{1} \mid D=\infty\right]} \text {, and } v \cdot \ell>0 .
$$

We are now ready to begin the derivation of some auxiliary estimates.

Lemma 1.1. There exists $\theta(\epsilon)>0$, such that for any connected $U \varsubsetneqq \mathbb{Z}^{d}$, containing 0 , and $x \in U \cup \partial U$,

$$
\exp \left\{-\theta X_{n} \cdot \ell\right\} \text { is an }\left(\mathcal{F}_{n}\right) \text {-supermartingale under } \widehat{P}_{x, U} .
$$

There exists $c_{1}(\epsilon)>0$, such that:

$$
P_{0}[D=\infty] \geq c_{1} .
$$


Proof. We begin with the proof of (1.22). We can find a numerical constant $\gamma>0$, such that for $\theta \in[0,1],|u| \leq 1$ :

$$
\left|e^{-\theta u}-1+\theta u\right| \leq \gamma \theta^{2} .
$$

Then for $n \geq 0, U$ as above and $x \in U$ :

$$
\begin{aligned}
& \widehat{E}_{x, U}\left[\exp \left\{-\theta X_{n+1} \cdot \ell\right\} \mid \mathcal{F}_{n}\right]=\exp \left\{-\theta X_{n} \cdot \ell\right\}\left(1\left\{X_{n} \in \partial U\right\}\right. \\
& \left.+1\left\{X_{n} \in U\right\} \widehat{E}_{X_{n}, U}\left[\exp \left\{-\theta\left(X_{1}-X_{0}\right) \cdot \ell\right\}\right]\right), \widehat{P}_{x, U} \text {-a.s. . }
\end{aligned}
$$

In view of Kalikow's condition (1.7), for $y \in U$,

$$
\widehat{E}_{y, U}\left[\exp \left\{-\theta\left(X_{1}-X_{0}\right) \cdot \ell\right\}\right] \leq 1-\theta \widehat{d}_{U}(y) \cdot \ell+\gamma \theta^{2} \leq 1,
$$

if $\theta \leq \theta(\epsilon)$. As a result when $\theta \leq \theta(\epsilon)$,

$$
\widehat{E}_{x, U}\left[\exp \left\{-\theta X_{n+1} \cdot \ell\right\} \mid \mathcal{F}_{n}\right] \leq \exp \left\{-\theta X_{n} \cdot \ell\right\}, \text { for } n \geq 0, \widehat{P}_{x, U} \text {-a.s. },
$$

and our claim (1.22) readily follows. We shall now write $\theta$ for $\theta(\epsilon)$.

We now turn to the proof of (1.23). We consider $M \geq 2 \sqrt{d}$, and the strip

$$
\mathcal{S}_{M}=\left\{x \in \mathbb{Z}^{d}, 0 \leq x \cdot \ell \leq M\right\} .
$$

This set is connected as easily follows from the fact (proven by connectedness) that the collection of vertices of closed unit cubes $z+[0,1]^{d}, z \in \mathbb{Z}^{d}$, intersecting a real line of $\mathbb{R}^{d}$ is a connected subset of $\mathbb{Z}^{d}$. Moreover $T_{\mathcal{S}_{M}}$ is finite $\widehat{P}_{0, \mathcal{S}_{M}}$-a.s.; indeed $X_{n} \cdot \ell$ is the sum of a martingale with bounded increments, which converges to a finite limit or oscillates between $+\infty$ and $-\infty$ on a set of full measure, see Durrett [5], p. 207, and an increasing process which tends to infinity on $\left\{T_{\mathcal{S}_{M}}=\infty\right\}$, in view of (1.7), (1.10). We can thus apply (1.6), and find:

$$
P_{0}\left[X_{T_{\mathcal{S}_{M}}} \cdot \ell>M\right]=\widehat{P}_{0, \mathcal{S}_{M}}\left[X_{T_{\mathcal{S}_{M}}} \cdot \ell>M\right] .
$$

The proof of (1.23) now involves a minor difficulty, which prevents the direct use of (1.22). Namely for certain $\ell$ the values of $\ell \cdot x$, with $x \in \mathbb{Z}^{d}$ such that $x \cdot \ell<0$, may accumulate in 0 . This fact is however easily overcome. Applying (1.22) and the stopping theorem, we see that:

$$
\begin{aligned}
\widehat{E}_{0, \mathcal{S}_{M}}[ & \left.X_{1} \cdot \ell \geq \frac{\epsilon}{2}, \exp \left\{-\theta X_{1} \cdot \ell\right\}\right] \\
& \geq \widehat{E}_{0, \mathcal{S}_{M}}\left[\exp \left\{-\theta X_{T_{\mathcal{S}_{M}}} \cdot \ell\right\}, X_{1} \cdot \ell \geq \frac{\epsilon}{2}\right] \\
& \geq \widehat{P}_{0, \mathcal{S}_{M}}\left[X_{1} \cdot \ell \geq \frac{\epsilon}{2}, X_{T_{\mathcal{S}_{M}}} \cdot \ell<0\right], \text { and therefore, }
\end{aligned}
$$




$$
\begin{aligned}
\widehat{P}_{0, \mathcal{S}_{M}}\left[X_{1} \cdot \ell \geq \frac{\epsilon}{2}, \quad X_{T_{\mathcal{S}_{M}}} \cdot \ell>M\right] \\
\quad \geq\left(1-\exp \left\{-\theta \frac{\epsilon}{2}\right\}\right) \widehat{P}_{0, \mathcal{S}_{M}}\left[X_{1} \cdot \ell \geq \frac{\epsilon}{2}\right] .
\end{aligned}
$$

Applying to $\left(X_{1} \cdot \ell\right)_{+}$the inequality

$$
P\left[X \geq \frac{1}{2} E[X]\right] \geq \frac{1}{4} \frac{E[X]^{2}}{E\left[X^{2}\right]},
$$

valid for $X$ a non-negative random variable with finite positive second moment, and using (1.7), we find:

$$
\widehat{P}_{0, \mathcal{S}_{M}}\left[X_{1} \cdot \ell \geq \frac{\epsilon}{2}\right] \geq \frac{1}{4} \epsilon^{2},
$$

and therefore in view of (1.28),

$$
P_{0}[D=\infty]=\lim _{M \rightarrow \infty} P_{0}\left[X_{T_{\mathcal{S}_{M}}} \cdot \ell>M\right] \geq \frac{1}{4} \epsilon^{2}\left(1-e^{-\theta \frac{\epsilon}{2}}\right),
$$

proving (1.23).

The next lemma shows that $X_{\tau_{1}} \cdot \ell$ has some finite exponential moment under $P_{0}$.

Lemma 1.2. There exist positive constants $c_{2}(d, \epsilon), c_{3}(d, \epsilon)$, such that:

$$
E_{0}\left[\exp \left\{c_{2} X_{\tau_{1}} \cdot \ell\right\}\right]<c_{3} .
$$

Proof. For $c>0$, thanks to (1.18):

$$
E_{0}\left[\exp \left\{c X_{\tau_{1}} \cdot \ell\right\}\right]=\sum_{k \geq 1} E_{0}\left[\exp \left\{c X_{S_{k}} \cdot \ell\right\}, S_{k}<\infty, D \circ \theta_{S_{k}}=\infty\right],
$$

and using the strong Markov property, when $k \geq 1$,

$$
\begin{aligned}
& E_{0}\left[\exp \left\{c X_{S_{k}} \cdot \ell\right\}, S_{k}<\infty, D \circ \theta_{S_{k}}=\infty\right]= \\
& \sum_{x \in \mathbb{Z}^{d}} \mathbb{E}\left[E_{0, \omega}\left[\exp \left\{c X_{S_{k}} \cdot \ell\right\}, S_{k}<\infty, X_{S_{k}}=x\right] P_{x, \omega}[D=\infty]\right]
\end{aligned}
$$

The terms under the above $\mathbb{E}$-expectation are respectively $\sigma(\omega(y, \cdot) ; \ell \cdot y<$ $\ell \cdot x)$ and $\sigma(\omega(y, \cdot) ; \ell \cdot y \geq \ell \cdot x)$-measurable, and thus $\mathbb{P}$-independent. As a result the above expression equals

$$
E_{0}\left[\exp \left\{c X_{S_{k}} \cdot \ell\right\}, S_{k}<\infty\right] P_{0}[D=\infty] \text {, }
$$


and we see that

$$
E_{0}\left[\exp \left\{c X_{\tau_{1}} \cdot \ell\right\}\right]=\sum_{k \geq 1} E_{0}\left[\exp \left\{c X_{S_{k}} \cdot \ell\right\}, S_{k}<\infty\right] P_{0}[D=\infty]
$$

$$
\begin{aligned}
& \stackrel{(1.15)}{\leq} \sum_{k \geq 1} E_{0}\left[\exp \left\{c X_{S_{k-1}} \cdot \ell\right\}, S_{k-1}<\infty,\right. \\
& \left.\exp \left\{c\left(a^{\prime}+M_{k-1}-X_{S_{k-1}} \cdot \ell\right)\right\}, D \circ \theta_{S_{k-1}}<\infty\right] P_{0}[D=\infty],
\end{aligned}
$$

where we use the definition below (1.15), the tacit convention that for an event $A$, " , $A$ " or ", $A$," inside an expectation stands for the indicator function of $A$, and the notation

$$
a^{\prime} \stackrel{\text { def }}{=} a+1 \stackrel{(1.14)}{\leq} 1+10 \sqrt{d} .
$$

Using a similar argument as after (1.33), the above equals:

$$
\begin{gathered}
\left(e^{c a^{\prime}}+\sum_{k \geq 2} E_{0}\left[\exp \left\{c X_{S_{k-1}} \cdot \ell\right\},\right.\right. \\
\left.\left.S_{k-1}<\infty\right] E_{0}\left[\exp \left\{c\left(a^{\prime}+\bar{M}\right)\right\}, D<\infty\right]\right) \\
\times P_{0}[D=\infty],
\end{gathered}
$$

provided we define

$$
\bar{M}=\sup \left\{X_{n} \cdot \ell, 0 \leq n \leq D\right\} .
$$

Using induction separately on each term of the above series, we thus see that:

$$
\begin{aligned}
& E_{0}\left[\exp \left\{c X_{\tau_{1}} \cdot \ell\right\}\right] \leq \\
& \quad e^{c a^{\prime}} P_{0}[D=\infty] \sum_{k \geq 1} E_{0}\left[\exp \left\{c\left(a^{\prime}+\bar{M}\right)\right\}, D<\infty\right]^{k-1} .
\end{aligned}
$$

The claim (1.32) will follow once we show that for $c_{2}(d, \epsilon)>0,0<$ $c_{4}(d, \epsilon)<1$,

$$
e^{c_{2}(1+10 \sqrt{d})} E_{0}\left[\exp \left\{c_{2} \bar{M}\right\}, D<\infty\right]<1-c_{4} .
$$

Observe that for $c>0$,

$$
\begin{gathered}
E_{0}[\exp \{c \bar{M}\}, \quad D<\infty] \leq \\
\quad \sum_{m \geq 0} e^{c 2^{m+1}} P_{0}\left[2^{m} \leq \bar{M}<2^{m+1}, D<\infty\right] \\
+e^{c} P_{0}[0 \leq \bar{M}<1, D<\infty] .
\end{gathered}
$$

Denote by $O_{m}$ and $\widetilde{O}_{m}$ the sets

$$
O_{m}=\left\{x \in \mathbb{Z}^{d}, \ell \cdot x<2^{m}\right\}, \widetilde{O}_{m}=\left\{x \in \mathbb{Z}^{d},|\ell \cdot x|<2^{m}\right\},
$$


so that $O_{m}$ is connected for $m \geq 0$, and $\widetilde{O}_{m}$ is connected when $2 \sqrt{d}<2^{m}$, see below (1.25). Introduce in analogy with $(0.5)$,

$$
\widetilde{T}_{u}=\inf \left\{n \geq 0, X_{n} \cdot \ell \leq u\right\}, \text { for } u \in \mathbb{R} .
$$

We can then proceed as in (2.16)-(2.25) of [25], and write for $m \geq 0$,

$$
\begin{array}{r}
P_{0}\left[2^{m} \leq \bar{M}<2^{m+1}, D<\infty\right] \leq P_{0}\left[\left|X_{T_{2^{m}}}-2^{m} \ell\right| \geq \frac{2^{m+1}}{\epsilon}\right] \\
+c(d)\left(\frac{2^{m+1}}{\epsilon}\right)^{d} P_{0}\left[\widetilde{T}_{-2^{m}}<T_{2^{m}}\right] .
\end{array}
$$

Moreover, on the event $\left\{\left|X_{T_{2^{m}}}-2^{m} \ell\right| \geq \frac{2^{m+1}}{\epsilon}\right\}$, we see that for $m \geq 1$,

$$
T_{O_{m}} \geq \frac{2^{m+1}}{\epsilon}, \widehat{P}_{0, O_{m}} \text {-a.s. . }
$$

Using (1.6), it follows that for $m \geq 1$,

$$
P_{0}\left[\left|X_{T_{2^{m}}}-2^{m} \ell\right| \geq \frac{2^{m+1}}{\epsilon}\right] \leq \widehat{P}_{0, O_{m}}\left[T_{O_{m}} \geq \frac{2^{m+1}}{\epsilon}\right]
$$

and since on $\left\{T_{O_{m}} \geq \frac{2^{m+1}}{\epsilon}\right\}$, Kalikow's condition (1.7) implies that:

$$
M_{\left[\frac{2^{m+1}}{\epsilon}\right]}^{O_{m}} \cdot \ell \leq-2^{m}+\epsilon \leq-2^{m-1},
$$

we find in view of Azuma's inequality (1.11), that for $m \geq 1$ :

$$
\begin{aligned}
P_{0}\left[\left|X_{T_{2^{m}}}-2^{m} \ell\right| \geq \frac{2^{m+1}}{\epsilon}\right] & \leq \widehat{P}_{0, O_{m}}\left[M_{\left[\frac{2^{m+1}}{\epsilon}\right]}^{O_{m}} \cdot \ell \leq-2^{m-1}\right] \\
& \leq \exp \left\{-\epsilon 2^{m-6}\right\} .
\end{aligned}
$$

Moreover, observing that $P_{0}$-a.s., $\left\{\widetilde{T}_{-2^{m}}<T_{2^{m}}\right\}=\left\{X_{\widetilde{O}_{m}} \cdot \ell \leq-2^{m}\right\}$, we see that when $2 \sqrt{d}<2^{m}$, the stopping theorem applied to the supermartingale in (1.22) and (1.6) imply that:

$$
P_{0}\left[\widetilde{T}_{-2^{m}}<T_{2^{m}}\right]=\widehat{P}_{0, \widetilde{o}_{m}}\left[X_{\widetilde{o}_{m}} \cdot \ell \leq-2^{m}\right] \stackrel{(1.22)}{\leq} \exp \left\{-\theta(\epsilon) 2^{m}\right\}
$$

However since in view of (1.23), $P_{0}[D<\infty] \leq 1-c_{1}(\epsilon)$, (1.39) together with the upperbounds (1.43), (1.44) on the terms entering the right hand side of (1.41), easily show (1.38). This proves our claim. 
The next lemma will be recurrently used when deriving upper bounds on the tail of $\tau_{1}$ in Sect. II and III. We first need some notations. We denote by $\left(e_{i}\right)_{i \in[1, d]}$, the canonical basis of $\mathbb{R}^{d}$, and choose some rotation $R$ of $\mathbb{R}^{d}$ such that:

$$
R\left(e_{1}\right)=\ell .
$$

For $L>0$, we denote by $C_{L}$ the cube:

$$
C_{L}=R\left((-L, L) \times\left(-\frac{2 L}{\epsilon}, \frac{2 L}{\epsilon}\right)^{d-1}\right) \cap \mathbb{Z}^{d},
$$

(recall $\epsilon$ is defined in (1.7)) .

Lemma 1.3. There exists $c_{5}(d, \epsilon)>0$, such that for any function $u \rightarrow$ $L(u)>0$, with $\lim _{u \rightarrow \infty} L(u)=\infty$,

$$
P_{0}\left[\tau_{1}>u\right] \leq P_{0}\left[T_{C_{L(u)}}=T_{L(u)}>u\right]+e^{-c_{5} L(u)},
$$

for large $u$, (see (0.5) for the notation) .

Proof. Keeping in mind (1.32), for $L(\cdot)$ as above, we find as an application of Chebyshev inequality:

$$
P_{0}\left[\tau_{1}>u\right] \leq P_{0}\left[\tau_{1}>u, X_{\tau_{1}} \cdot \ell \leq L(u)\right]+e^{-c_{2} L(u)} E_{0}\left[e^{c_{2} X_{\tau_{1}} \cdot \ell}\right] .
$$

By the very construction of $\tau_{1}$, cf. (1.15)-(1.18), $\tau_{1}=T_{X_{\tau_{1}} \cdot \ell}$, and thus for large $u$,

$$
P_{0}\left[\tau_{1}>u\right] \leq P_{0}\left[T_{L(u)}>u\right]+\exp \left\{-\frac{c_{2}}{2} L(u)\right\} .
$$

Moreover,

$$
P_{0}\left[T_{L(u)}>u\right]=P_{0}\left[T_{C_{L(u)}}=T_{L(u)}>u\right]+P_{0}\left[T_{L(u)}>T_{C_{L(u)}}\right] .
$$

If $\widetilde{C}$ denotes the connected component of 0 in $C_{L(u)}$, then $\partial \widetilde{C} \subseteq \partial C_{L(u)}$, and using (1.6),

$$
\begin{aligned}
& P_{0}\left[T_{L(u)}>T_{C_{L(u)}}\right]=\widehat{P}_{0, \widetilde{C}}\left[X_{T_{\widetilde{C}}} \cdot \ell<L(u)\right] \leq \\
& \widehat{P}_{0, \widetilde{C}}\left[T_{\widetilde{C}}>\frac{2}{\epsilon} L(u)\right]+\widehat{P}_{0, \widetilde{C}}\left[T_{\widetilde{C}} \leq \frac{2}{\epsilon} L(u), X_{T_{\widetilde{C}}} \cdot \ell<L(u)\right] .
\end{aligned}
$$

Introducing $N=\left[\frac{2}{\epsilon} L(u)\right]$, as after (1.42), for large $u$,

$$
\begin{aligned}
\widehat{P}_{0, \widetilde{C}}\left[T_{\widetilde{C}} \geq \frac{2}{\epsilon} L(u)\right] & \leq \widehat{P}_{0, \widetilde{C}}\left[M_{N}^{\widetilde{C}} \cdot \ell \leq-L(u)+\epsilon\right] \\
& (1.11) \exp \left\{-\frac{L^{2}(u)}{16 N}\right\} .
\end{aligned}
$$


By the same argument as after (1.42),

$$
\begin{aligned}
& \widehat{P}_{0, \widetilde{C}}\left[T_{\widetilde{C}}<\frac{2}{\epsilon} L(u), X_{T_{\widetilde{C}}} \cdot \ell<L(u)\right] \leq \widehat{P}_{0, \widetilde{C}}\left[X_{T_{\widetilde{C}}} \cdot \ell \leq-L(u)\right] \\
& \stackrel{(1.22)}{\leq} \exp \{-\theta(\epsilon) L(u)\} .
\end{aligned}
$$

Therefore combining (1.51) and (1.52), we see that for large $u$,

$$
P_{0}\left[T_{L(u)}>T_{C_{L(u)}}\right] \leq \exp \left\{-\frac{L^{2}(u)}{16 N}\right\}+\exp \{-\theta(\epsilon) L(u)\},
$$

and coming back to (1.48), (1.49), our claim follows.

We shall now prove a reinforcement of Lemma 1.2, which will play an important role in the control of fluctuations of the walk transversal to the direction of the limiting velocity. This will in particular come into play in Sect. III, when deriving upper bounds on the tail of $\tau_{1}$, for $d \geq 2$. We first define

$$
X_{*}=\sup \left\{\left|X_{n}-X_{0}\right|, 0 \leq n \leq \tau_{1}\right\} .
$$

Proposition 1.4. There exist positive constants $c_{6}(d, \epsilon), c_{7}(d, \epsilon)$ such that

$$
E_{0}\left[\exp \left\{c_{6} X_{*}\right\}\right] \leq c_{7} .
$$

Proof. In view of Lemma 1.2, it suffices to prove a statement analogous to (1.55) with $X_{*}$ replaced by

$$
X_{*}(u)=\sup \left\{\left(X_{n}-X_{0}\right) \cdot u, 0 \leq n \leq \tau_{1}\right\},
$$

with $u=-\ell$, or $u=R\left( \pm e_{i}\right), 2 \leq i \leq d$, in the notation of (1.45).

The case of $u=-\ell$ is easily taken care of, with the help of the estimate

$$
P_{0}\left[\widetilde{T}_{-2^{m}}<\infty\right] \leq \exp \left\{-\theta(\epsilon) 2^{m}\right\}, m \geq 0,
$$

as follows from (1.6) and (1.22), just as in (1.44). One then simply uses the inequality:

$E_{0}\left[\exp \left\{c X_{*}(-\ell)\right\}\right] \leq e^{c}+\sum_{m \geq 0} e^{c 2^{m+1}} P_{0}\left[\widetilde{T}_{-2^{m}}<\infty, \widetilde{T}_{-2^{m+1}}=\infty\right]$, for $c>0$,

and concludes from (1.56) that the left hand side is finite for small $c(\epsilon)$. 
We thus suppose from now on that $u=R\left( \pm e_{i}\right)$, with $i \in[2, d]$. For $c>0$, we have:

$$
\begin{aligned}
& E_{0}\left[\exp \left\{c X_{*}(u)\right\}, X_{*}(u)>\frac{4}{\epsilon} X_{\tau_{1}} \cdot \ell\right] \leq e^{\frac{2 c}{\epsilon}} \\
& +\sum_{m^{\prime} \geq 0} E_{0}\left[\exp \left\{c X_{*}(u)\right\}, X_{\tau_{1}} \cdot \ell<2^{m^{\prime}}, \frac{2^{m^{\prime}+1}}{\epsilon} \leq X_{*}(u)\right] \leq e^{\frac{2 c}{\epsilon}} \\
& +\sum_{m>m^{\prime} \geq 0} e^{\frac{c 2^{m+1}}{\epsilon}} P_{0}\left[X_{*}(u) \geq \frac{2^{m}}{\epsilon}, X_{\tau_{1}} \cdot \ell<2^{m^{\prime}}\right] .
\end{aligned}
$$

If we now introduce for $0 \leq m^{\prime}<m$ :

$$
U=\left\{x \in \mathbb{Z}^{d} ; x \cdot u<\frac{2^{m}}{\epsilon}, x \cdot \ell<2^{m^{\prime}}\right\},
$$

and denote by $\widetilde{U}$ the connected component of 0 in $U$, so that $\partial \widetilde{U} \subseteq \partial U$, using the fact that $\tau_{1}=T_{X_{\tau_{1}}} \cdot \ell$, we see just as in (1.50) that for $0 \leq m^{\prime}<m$ :

$$
\begin{aligned}
& P_{0}\left[X_{*}(u) \geq \frac{2^{m}}{\epsilon}, X_{\tau_{1}} \cdot \ell<2^{m^{\prime}}\right] \leq \\
& P_{0}\left[T_{\widetilde{U}}<T_{2^{m^{\prime}}}\right]=\widehat{P}_{0, \widetilde{U}}\left[X_{T_{\widetilde{U}}} \cdot \ell<2^{m^{\prime}}, X_{T_{\widetilde{U}}} \cdot u \geq \frac{2^{m}}{\epsilon}\right] \leq \\
& \widehat{P}_{0, \widetilde{U}}\left[T_{\widetilde{U}} \geq \frac{2^{m}}{\epsilon}\right]+\widehat{P}_{0, \widetilde{U}}\left[T_{\widetilde{U}}<\frac{2^{m}}{\epsilon}, X_{T_{\widetilde{U}}} \cdot u \geq \frac{2^{m}}{\epsilon}\right] .
\end{aligned}
$$

Then by a similar argument as after (1.42), the rightmost term vanishes, and setting $N=\left[\frac{2^{m+1}}{\epsilon}\right]$, we find that the above expression is smaller than:

$$
\exp \left\{-\frac{\left(2^{m-1}-1\right)^{2}}{8 N}\right\}
$$

As a result, we see that for $c>0$,

$$
\begin{aligned}
& E_{0}\left[\exp \left\{c X_{*}(u)\right\}\right] \leq E_{0}\left[\exp \left\{\frac{4 c}{\epsilon} X_{\tau_{1}} \cdot \ell\right\}\right]+e^{\frac{2 c}{\epsilon}}+ \\
& \sum_{m \geq 1} m e^{\frac{c 2^{m+1}}{\epsilon}} \exp \left\{-\frac{\left(2^{m-1}-1\right)^{2}}{8 N}\right\} .
\end{aligned}
$$

Our claim (1.55) now easily follows.

We shall now spell out some consequences of the above proposition in the next

Corollary 1.5. For $\gamma \in\left(\frac{1}{2}, 1\right]$ and $\rho>0$,

$$
\varlimsup_{u \rightarrow \infty} u^{1-2 \gamma} \log P_{0}\left[\sup _{0 \leq n \leq L_{u}}\left|\pi\left(X_{n}\right)\right|>\rho u^{\gamma}\right]<0,
$$


where $L_{u}=\sup \left\{n \geq 0, X_{n} \cdot \ell \leq u\right\}$, stands for the last visit of $X_{n}$ to $\left\{x \in \mathbb{Z}^{d}, \ell \cdot x \leq u\right\}$, and $\pi(z)=z-\frac{z \cdot v}{|v|^{2}} v$, denotes the orthogonal projection on the orthogonal subspace of $v$. Moreover, if $z \notin \mathbb{R}_{+} v(=\{\lambda v, \lambda \geq 0\})$,

$$
\varlimsup_{n \rightarrow \infty} \frac{1}{n} \log P_{0}\left[H_{[n z]}<\infty\right]<0,
$$

where $[n z]$ denotes a closest point in $\mathbb{Z}^{d}$ to $n z$.

Proof. We begin with the proof of (1.60). Observe that without loss of generality we can replace $\left|\pi\left(X_{n}\right)\right|$ in (1.60) by $X_{n} \cdot w$, where $w \in \mathbb{R}^{d}$ is such that $w \cdot v=0$. Let us define for $n \geq 1$,

$$
K_{n}=\sup \left\{k \geq 0, \tau_{k}<n\right\},
$$

(see the notation below (1.18)). Since $P_{0}$-a.s., for $k \geq 1, X_{m} \cdot \ell \geq X_{\tau_{k}} \cdot \ell \geq k a$, for $m \geq \tau_{k}$, we see that

$$
P_{0} \text {-a.s., for } u>0,0 \leq n \leq L_{u} \text {, implies } K_{n} \leq \frac{u}{a} .
$$

Thus for $w$ as in (1.60), we see that $P_{0}$-a.s., for $n \geq 1$,

$$
X_{n} \cdot w=X_{\tau_{K_{n}}} \cdot w+\left(X_{n}-X_{\tau_{K_{n}}}\right) \cdot w \leq X_{\tau_{K_{n}}} \cdot w+X_{*} \circ \theta_{\tau_{K_{n}}} .
$$

As a result, for $\rho>0, \gamma \in\left(\frac{1}{2}, 1\right]$ and $u>0$ :

$$
\begin{aligned}
& P_{0}\left[\sup _{0 \leq n \leq L_{u}} X_{n} \cdot w>\rho u^{\gamma}\right] \leq \sum_{0 \leq k \leq \frac{u}{a}} P_{0}\left[X_{\tau_{k}} \cdot w+X_{*} \circ \theta_{\tau_{k}}>\rho u^{\gamma}\right] \\
& \leq \sum_{0 \leq k \leq \frac{u}{a}} P_{0}\left[X_{*} \circ \theta_{\tau_{k}}>\frac{\rho}{3} u^{\gamma}\right] \\
& \quad+\sum_{1 \leq k \leq \frac{u}{a}}\left(P_{0}\left[X_{\tau_{1}} \cdot w>\frac{\rho}{3} u^{\gamma}\right]+P_{0}\left[\left(X_{\tau_{k}}-X_{\tau_{1}}\right) \cdot w>\frac{\rho}{3} u^{\gamma}\right]\right) .
\end{aligned}
$$

Using now (1.19), together with Chebyshev's inequality, we see that for $\lambda>0$, the above is smaller than

$$
\begin{aligned}
\exp \left\{-\frac{\lambda \rho}{3} u^{\gamma}\right\}\left(\left(\frac{2 u}{a}+1\right) E_{0}[\right. & \left.\exp \left\{\lambda X_{*}\right\}\right] P_{0}[D=\infty]^{-1}+ \\
& \left.\sum_{1 \leq k \leq \frac{u}{a}} E_{0}\left[\exp \left\{\lambda X_{\tau_{1}} \cdot w\right\} \mid D=\infty\right]^{k-1}\right) .
\end{aligned}
$$

Introduce for $|\lambda|<c_{6}$, the convex function

$$
H(\lambda)=\log E_{0}\left[\exp \left\{\lambda X_{\tau_{1}} \cdot w\right\} \mid D=\infty\right] .
$$

Since $E_{0}\left[X_{\tau_{1}} \cdot u \mid D=\infty\right]=0$, we see that $H(0)=0, H^{\prime}(0)=0, H(\cdot) \geq 0$ for $\lambda \geq 0$, and $H(\lambda)=O\left(\lambda^{2}\right)$, as $\lambda \rightarrow 0$. 
Moreover, the above shows that for $\lambda \in\left(0, c_{6}\right)$ and $u>0$ :

$$
\begin{aligned}
& P_{0}\left[\sup _{0 \leq n \leq L_{u}} X_{n} \cdot w>\rho u^{\gamma}\right] \leq\left(\frac{2 u}{a}+1\right) \exp \left\{-\frac{\lambda}{3} \rho u^{\gamma}\right\} \\
& \left(E_{0}\left[\exp \left\{\lambda X_{*}\right\}\right] P_{0}[D=\infty]^{-1}+\exp \left\{\frac{u}{a} H(\lambda)\right\}\right) .
\end{aligned}
$$

When $\gamma=1$, choosing $\lambda \in\left(0, c_{6}\right)$ small enough so that $H(\lambda)<\frac{\lambda}{3} \rho a$, we thus complete the proof of (1.60). In the case $\gamma \in\left(\frac{1}{2}, 1\right)$, we instead choose for a sufficiently small $v>0, \lambda=\left(v u^{\gamma-1}\right) \wedge c_{6}$, and conclude in a similar fashion.

Let us now turn to the proof of (1.61). When $z=-\lambda v, \lambda>0$, the claim easily follows from (1.56), and we only need consider the case

$$
z \notin \mathbb{R} v
$$

Observe that

(1.67) $P_{0}$-a.s., on $\left\{H_{[n z]}<\infty\right\}, H_{[n z]} \leq L_{c_{8} n}$, where $c_{8}=|z \cdot \ell|+\sqrt{d}$.

With the help of (1.66) we can choose a unit vector $w$ with $w \cdot v=0$, and $w \cdot z>0$. We thus have for $n \geq 1$ :

$$
P_{0} \text {-a.s., on }\left\{H_{[n z]}<\infty\right\}, \sup _{0 \leq k \leq L_{c g^{n}}}\left|\pi\left(X_{k}\right)\right| \geq n z \cdot w-\sqrt{d}
$$

so that the claim (1.61) follows from (1.60).

The above corollary has a direct consequence on the structure of the null set of the 0-th (quenched) Lyapunov coefficient of Zerner [26]. It is shown in Theorem A of [26], that under assumptions which are implied by $(0.1)$, on a set of full $\mathbb{P}$-measure,

$$
\begin{aligned}
& \text { for all } \mathbb{Z}^{d} \text {-valued sequences }\left(y_{n}\right)_{n \geq 0} \text { tending to infinity, } \\
& \lim _{n} \frac{1}{\left|y_{n}\right|}\left(\log P_{0, \omega}\left[H_{y_{n}}<\infty\right]+\alpha_{0}\left(y_{n}\right)\right)=0,
\end{aligned}
$$

where $\alpha_{0}(\cdot)$ is a deterministic, non-negative, convex, homogeneous function of degree 1 , on $\mathbb{R}^{d}$, (which is in particular continuous). The structure of the null set of $\alpha_{0}(\cdot)$ is in general poorly understood, however in our present setting, we have

Proposition 1.6. Under (0.1) and (1.7),

$$
\alpha_{0}(w)=0 \Longleftrightarrow w \in \mathbb{R}_{+} v \text {. }
$$


Proof. From a Borel-Cantelli type argument and (1.61), we see that when $w \notin \mathbb{R}_{+} v$ :

$$
\mathbb{P} \text {-a.s., } \varlimsup_{n} \frac{1}{n} \log P_{0, \omega}\left[H_{[n w]}<\infty\right]<0,
$$

and as a result of (1.69), $\alpha_{0}(w)>0$. On the other hand, $\alpha_{0}(w)$ must vanish for some $|w|=1$, otherwise setting $\alpha_{*}=\inf \left\{\alpha_{0}(w),|w|=1\right\}$, (1.69) implies that $\mathbb{P}$-a.s., except for finitely many $x, P_{0, \omega}\left[H_{x}<\infty\right] \leq e^{-\frac{\alpha *|x|}{2}}$, which would imply that $P_{0}\left[\sup _{n}\left|X_{n}\right|<\infty\right]=1$. Thus $\alpha_{0}(w)=0$, for some $w=\lambda v$, with $\lambda>0$, and thus $\alpha_{0}(w)=0$, for $\omega \in \mathbb{R}_{+} v$, since $\alpha_{0}$ is homogeneous. This completes the proof of (1.70).

\section{First tail estimates on the renewal time}

In this section we shall derive some first tail estimates on the variable $\tau_{1}$ in the non-nestling, marginal nestling and plain nestling situation, cf. (0.13). This will in particular highlight the differences between these three cases. The main result of this section however concerns the tail behavior of $\tau_{1}$ for walks which are neutral or biased to the right, (a marginal nestling case). Let us recall that Kalikow's condition relative to a suitable $\ell \in S^{d-1}$, i.e. (1.7), will hold in all examples we consider. We begin with the simpler case of

\section{A) Non-nestling walks}

As in the introduction, we denote by $K_{o}$ the convex hull of the support of the law of $d(0, \omega)$ (a compact subset of $\left.\mathbb{R}^{d}\right)$. We only consider $\ell \in S^{d-1}$, such that:

$$
\inf _{w \in K_{o}} w \cdot \ell \stackrel{\text { def }}{=} \eta>0
$$

in view of the non-nestling assumption, cf. (0.13) i), such $\ell$ exists, and Proposition 2.4 of [25] implies that Kalikow's condition (1.7) holds, (it is also a simple matter to check (1.7) directly, using (1.4)). In the present setting, as we shall now see, $\tau_{1}$ has an exponential tail:

Theorem 2.1. $(d \geq 1)$

$$
-\infty<\varliminf_{u \rightarrow \infty} \frac{1}{u} \log P_{0}\left[\tau_{1}>u\right] \leq \varlimsup_{u \rightarrow \infty} \frac{1}{u} \log P_{0}\left[\tau_{1}>u\right]<0 .
$$

Proof. The lower estimate is immediate since in view of the definition of $\tau_{1}$ in (1.18), for $u$ an even integer, 


$$
\begin{aligned}
P_{0}\left[\tau_{1}>u\right] & \geq P_{0}\left[X_{u}=0\right] \\
& \geq P_{0}\left[X_{2 j+1}=e_{1}, X_{2 j+2}=0,0 \leq j<\frac{u}{2}\right] \stackrel{(0.1)}{\geq} \kappa^{u},
\end{aligned}
$$

(see above (1.45) for the notation $e_{1}$ ).

As for the upper bound, in view of Lemma 1.3, we see that for large $u$ and $\eta$ as in (2.1):

$$
P_{0}\left[\tau_{1}>u\right] \leq \exp \left\{-c_{5} u \frac{\eta}{2}\right\}+P_{0}\left[T_{C_{u \frac{\eta}{2}}}>u\right],
$$

and for large $u$ and $\mathbb{P}$-a.e. $\omega$ :

$$
P_{0, \omega}\left[T_{C_{u \frac{\eta}{2}}}>u\right] \leq P_{0, \omega}\left[X_{[u]} \cdot \ell-\sum_{k=0}^{[u]-1} d\left(X_{k}, \omega\right) \cdot \ell \leq-\eta \frac{u}{3}\right] \leq \exp \left\{-\frac{\eta^{2}}{72} u\right\},
$$

using Azuma's inequality, cf. [1], p. 85, in the last step. Our claim follows.

We shall now turn to the marginal nestling and then plain nestling situation. In both cases the nestling property holds (i.e. $0 \in K$ ), and in view of Proposition 8 (I) of Zerner [26], it implies that regardless of $\ell$, in subsection $\mathrm{B}$ ) and $\mathrm{C}$ ) below:

$$
\varlimsup_{u} u^{-1} \log P_{0}\left[\tau_{1}>u\right] \geq \varlimsup_{n} n^{-1} \log P_{0}\left[X_{n}=0\right]=0,
$$

in contrast to (2.2).

\section{B) Marginal nestling case}

We assume now that $0 \in \partial K_{o}, \ell \in S^{d-1}$ is such that (1.7) holds and

$$
K_{o} \subseteq\left\{w \in \mathbb{R}^{d}, w \cdot \ell \geq 0\right\} .
$$

With the help of Proposition 2.4 of [25], these assumptions are equivalent to:

(2.5) $0 \in K_{o} \subseteq\left\{w \in \mathbb{R}^{d}, w \cdot \ell \geq 0\right\}$, and $K_{o} \cap\left\{w \in \mathbb{R}^{d}, w \cdot \ell>0\right\} \neq \emptyset$.

It is convenient to introduce

$$
\Omega_{+}=\left\{\omega \in \Omega: \forall x \in \mathbb{Z}^{d}, d(x, \omega) \cdot \ell \geq 0\right\},
$$

which has full $\mathbb{P}$-measure. The following lemma will be helpful.

Lemma 2.2. $(d \geq 1)$. There exists $c_{9}(\kappa)>0$ such that, for $L \geq 1, \omega \in \Omega_{+}$, $x \in \mathbb{Z}^{d}$,

$$
\begin{gathered}
E_{x, \omega}\left[\exp \left\{2 \frac{c_{9}}{L^{2}} T_{U_{L}}\right\}\right] \leq 2, \text { where } \\
U_{L}=\left\{y \in \mathbb{Z}^{d},|y \cdot \ell|<L\right\} .
\end{gathered}
$$


Proof. By a classical argument of Khas'minskii, see [10] for the original reference and for instance Lemma 1.1 of [24] for the present statement, it suffices to show that for some positive constant $c_{10}(\kappa)$ :

$$
E_{x, \omega}\left[T_{U_{L}}\right] \leq c_{10}(\kappa) L^{2}, \text { for } L \geq 1, \omega \in \Omega_{+}, x \in \mathbb{Z}^{d} .
$$

We assume from now on that $\omega \in \Omega_{+}$. Consider the non-decreasing process

$$
A_{n}=\sum_{0}^{n-1} d\left(X_{k}, \omega\right) \cdot \ell, n \geq 0,
$$

and the $P_{x, \omega}$-martingale:

$$
M_{n}=X_{n}-X_{0}-\sum_{0}^{n-1} d\left(X_{k}, \omega\right), n \geq 0 .
$$

The stopping theorem applied to $M_{n} \cdot \ell$ implies that

$$
E_{x, \omega}\left[A_{T_{U_{L}}}\right] \leq 2(L+1) \leq 4 L, \text { for } L \geq 1, x \in \mathbb{Z}^{d}, \omega \in \Omega_{+} .
$$

Moreover the process

$$
\begin{aligned}
\widetilde{M}_{n}=\left(X_{n} \cdot \ell\right)^{2} & -\left(X_{0} \cdot \ell\right)^{2} \\
& -\sum_{k=0}^{n-1} \sum_{|e|=1} \omega\left(X_{k}, e\right)\left[\left(X_{k} \cdot \ell+e \cdot \ell\right)^{2}-\left(X_{k} \cdot \ell\right)^{2}\right] \\
=\left(X_{n} \cdot \ell\right)^{2} & -\left(X_{0} \cdot \ell\right)^{2} \\
& -\sum_{k=0}^{n-1}\left[2 X_{k} \cdot \ell d\left(X_{k}, \omega\right) \cdot \ell+\sum_{|e|=1} \omega\left(X_{k}, e\right)(e \cdot \ell)^{2}\right]
\end{aligned}
$$

also defines a $P_{x, \omega}$-martingale.

A second application of the stopping theorem together with (2.11), and the inequalities

$\sum_{0}^{T_{U_{L}}-1} \sum_{|e|=1} \omega\left(X_{k}, e\right)(e \cdot \ell)^{2} \stackrel{(0.1)}{\geq} \kappa T_{U_{L}},\left|\sum_{0}^{T_{U_{L}}-1} 2 X_{k} \cdot \ell d\left(X_{k}, \omega\right) \cdot \ell\right| \leq 2 L A_{T_{U_{L}}}$, implies (2.9). Our claim follows.

We shall first infer a simple upper bound on the tail of $\tau_{1}$ in the following

Proposition 2.3. $(d \geq 1)$. Under (0.1) and (2.5),

$$
\varlimsup_{u \rightarrow \infty} u^{-1 / 3} \log P_{0}\left[\tau_{1}>u\right]<0 .
$$


Proof. Choosing $L(u)=u^{1 / 3}$, in Lemma 1.3, it suffices to prove that

$$
\varlimsup_{u} u^{-1 / 3} \log P_{0}\left[T_{C_{L(u)}}>u\right]<0 .
$$

However, when $L(u)>1$, using Chebyshev's inequality,

$P_{0}\left[T_{C_{L(u)}}>u\right] \leq P_{0}\left[T_{U_{L(u)}}>u\right] \stackrel{(2.7)}{\leq} \exp \left\{-\frac{2 c_{9}}{L(u)^{2}} u\right\} 2=2 \exp \left\{-2 c_{9} u^{1 / 3}\right\}$,

proving (2.14).

The above simple upper bound will in particular distinguish the present situation from the strongly-nestling case in C). It will also ensure sufficient integrability of $\tau_{1}$ to infer a central limit theorem for $B^{n}$, cf. Sect. IV. The next result will highlight the importance of certain large deviation estimates on the exit distribution of a large slab $U_{L}$, (see (2.8)).

Theorem 2.4. $(d \geq 1)$. Assume (0.1), (2.5) and suppose that for some $p_{0} \in\left(0, \frac{1}{2}\right)$ and $\alpha \in(1, \infty)$,

$$
\begin{gathered}
\varlimsup_{L \rightarrow \infty} L^{-\alpha} \log \mathbb{P}\left[P_{0, \omega}\left[X_{T_{U_{L}}} \cdot \ell \leq-L\right] \geq p_{0}\right]<0, \text { then } \\
\varlimsup_{u \rightarrow \infty} u^{-\frac{\alpha}{\alpha+2}} \log P_{0}\left[\tau_{1}>u\right]<0 .
\end{gathered}
$$

Proof. We introduce for $u>1$,

$$
L(u)=u^{\frac{1}{\alpha+2}} N(u), \text { with } N(u)=\left[u^{\frac{\alpha-1}{\alpha+2}}\right] .
$$

As a consequence of Lemma 1.3, our claim will follow from:

$$
\varlimsup_{u \rightarrow \infty} u^{-\frac{\alpha}{\alpha+2}} \log P_{0}\left[T_{L(u)}=T_{C_{L(u)}}>u\right]<0 .
$$

Consider the stopping time

$$
\bar{S}_{1}=\inf \left\{n \geq 0, \quad\left|X_{n} \cdot \ell-X_{0} \cdot \ell\right| \geq u^{\frac{1}{\alpha+2}}\right\},
$$

as well as its iterates $\bar{S}_{k}, k \geq 0$, such that

$$
\bar{S}_{0}=0, \quad \bar{S}_{k+1}=\bar{S}_{1} \circ \theta_{\bar{S}_{k}}+\bar{S}_{k}, \text { for } k \geq 1 .
$$

We can now define

(2.21) $\bar{T}_{1}=\bar{S}_{V}$, where $V=\inf \left\{k \geq 0, X_{\bar{S}_{k}} \cdot \ell-X_{0} \cdot \ell \geq u^{\frac{1}{\alpha+2}}\right\}$.

Note that when $\omega \in \Omega_{+}$, for any $x \in \mathbb{Z}^{d}, \overline{\lim }_{n} X_{n} \cdot \ell=\infty, P_{x, \omega}$-a.s. . Indeed $X_{n} \cdot \ell$ is the sum of a martingale with bounded increments and a non-decreasing process, moreover it does not have a finite limit, so that 
the claim follows from Durrett [5], p. 207 (see also after (1.25)). As a result when $\omega \in \Omega_{+}, V$ is finite $P_{x, \omega}$-a.s., for any $x \in \mathbb{Z}^{d}$. We can now define the iterates of $\bar{T}_{1}$ :

$$
\bar{T}_{0}=0, \bar{T}_{j+1}=\bar{T}_{1} \circ \theta_{\bar{T}_{j}}+\bar{T}_{j}, j \geq 1 .
$$

Observe also that in the notation of (0.5),

$$
\begin{gathered}
\bar{T}_{1}<T_{X_{0} \cdot \ell+2 u^{\frac{1}{\alpha+2}}+1}, \quad \text { so that } \\
P_{0} \text {-a.s., } T_{L(u)} \leq \bar{T}_{N(u)} \leq T_{3 L(u)},
\end{gathered}
$$

and in view of (1.53),

$$
\varlimsup_{u \rightarrow \infty} u^{-\frac{\alpha}{\alpha+2}} \log P_{0}\left[T_{3 L(u)} \geq T_{C_{4 L(u)}}\right]<0 .
$$

Our claim (2.18) will then follow if we show that

$$
\varlimsup_{u \rightarrow \infty} u^{-\frac{\alpha}{\alpha+2}} \log P_{0}\left[u<\bar{T}_{N(u)}<T_{C_{4 L(u)}}\right]<0 .
$$

Then consider for $u>1$, the event

$$
\mathcal{E}=\left\{\omega \in \Omega_{+}, \sup _{x \in C_{4 L}(u)} P_{x, \omega}\left[X_{\bar{S}_{1}} \cdot \ell \leq x \cdot \ell-u^{\frac{1}{\alpha+2}}\right] \leq p_{0}\right\},
$$

where $p_{0}$ appears in (2.15). In view of the polynomial growth of $\left|C_{4 L(u)}\right|$ in $u$, we see that:

$$
\varlimsup_{u \rightarrow \infty} u^{-\frac{\alpha}{\alpha+2}} \log \mathbb{P}\left[\mathcal{E}^{c}\right]<0,
$$

and (2.25) will thus follow once we show that

$$
\varlimsup_{u \rightarrow \infty} u^{-\frac{\alpha}{\alpha+2}} \log \sup _{\omega \in \mathcal{E}} P_{0, \omega}\left[u<\bar{T}_{N(u)}<T_{C_{4 L(u)}}\right]<0 .
$$

Now for $\lambda>0, \omega \in \mathcal{E}, u>1$,

$$
\begin{aligned}
& P_{0, \omega}\left[u<\bar{T}_{N(u)}<T_{C_{4 L(u)}}\right] \leq \\
& \exp \left\{-\lambda u^{\frac{\alpha}{\alpha+2}}\right\} E_{0, \omega}\left[\exp \left\{\lambda u^{-\frac{2}{\alpha+2}} \sum_{i=0}^{N-1} \bar{T}_{1} \circ \theta_{\bar{T}_{i}}\right\}, \bar{T}_{N}<T_{C_{4 L}}\right],
\end{aligned}
$$

writing for simplicity $N$ and $L$ in place of $N(u), L(u)$. Applying the strong Markov property, we find:

$$
\begin{aligned}
& E_{0, \omega}\left[\exp \left\{\lambda u^{-\frac{2}{\alpha+2}} \sum_{i=0}^{N-1} \bar{T}_{1} \circ \theta_{\bar{T}_{i}}\right\}, \bar{T}_{N}<T_{C_{4 L}}\right] \leq \\
& E_{0, \omega}\left[\exp \left\{\lambda u^{-\frac{2}{\alpha+2}} \sum_{i=0}^{N-2} \bar{T}_{1} \circ \theta_{\bar{T}_{i}}\right\}, \bar{T}_{N-1}<T_{C_{4 L}},\right. \\
& \left.E_{X_{\bar{T}_{N-1}}, \omega}\left[\exp \left\{\lambda u^{-\frac{2}{\alpha+2}} \bar{S}_{V}\right\}, \bar{S}_{V}<T_{C_{4 L}}\right]\right] .
\end{aligned}
$$


An application of Lemma 2.2 and Jensen's inequality implies that for $\omega \in$ $\Omega_{+}, u>1, x \in \mathbb{Z}^{d}, \lambda \in\left(0,2 c_{9}\right)$,

$$
\begin{aligned}
& E_{x, \omega}\left[\exp \left\{\lambda u^{-\frac{2}{\alpha+2}} \bar{S}_{1}-\psi(\lambda)\right\}\right] \leq 1, \\
& \text { with } \psi(\cdot):\left(0,2 c_{9}\right) \rightarrow \mathbb{R}_{+}, \text {such that } \lim _{\lambda \rightarrow 0} \psi(\lambda)=0 .
\end{aligned}
$$

As a result $\exp \left\{\lambda u^{-\frac{2}{\alpha+2}} \bar{S}_{k}-k \psi(\lambda)\right\}$ is an $\left(\mathcal{F}_{\bar{S}_{k}}\right)$-supermartingale under $P_{x, \omega}$, and the stopping theorem implies that for $\omega, u, x$, as above, and $\lambda \in\left(0, c_{9}\right)$ :

$$
E_{x, \omega}\left[\exp \left\{2 \lambda u^{-\frac{2}{\alpha+2}} \bar{S}_{V}-\psi(2 \lambda) V\right\}\right] \leq 1 .
$$

Applying Cauchy-Schwarz's inequality, we see that for $u \geq 1, \omega \in \mathcal{E}$, $x \in C_{4 L}, \lambda \in\left(0, c_{9}\right)$ :

$$
\begin{aligned}
& E_{x, \omega}\left[\exp \left\{\lambda u^{-\frac{2}{\alpha+2}} \bar{S}_{V}\right\}, \bar{S}_{V}<T_{C_{4 L}}\right] \leq \\
& E_{x, \omega}\left[\exp \{\psi(2 \lambda) V\}, \bar{S}_{V}<T_{C_{4 L}}\right]^{1 / 2} .
\end{aligned}
$$

Furthermore, when $x \in C_{4 L}$, for $\kappa_{1}, \kappa_{2}>0$, and $k \geq 0$, the strong Markov property implies that:

$$
\begin{aligned}
& E_{x, \omega}\left[\exp \left\{\kappa_{1}[(k+1) \wedge V]-\kappa_{2} u^{-\frac{1}{\alpha+2}} X_{\bar{S}_{(k+1) \wedge V}} \cdot \ell\right\}, \bar{S}_{(k+1) \wedge V}<T_{C_{4 L}}\right] \leq \\
& E_{x, \omega}\left[k \geq V, \bar{S}_{k \wedge V}<T_{C_{4 L}}, \exp \left\{\kappa_{1}(k \wedge V)-\kappa_{2} u^{-\frac{1}{\alpha+2}} X_{\bar{S}_{k \wedge V}} \cdot \ell\right\}\right]+ \\
& E_{x, \omega}\left[k<V, \bar{S}_{k \wedge V}<T_{C_{4 L}}, \exp \left\{\kappa_{1} k-\kappa_{2} u^{-\frac{1}{\alpha+2}} X_{\bar{S}_{k}} \cdot \ell\right\},\right. \\
& \left.E_{X_{\bar{S}_{k}}, \omega}\left[\exp \left\{\kappa_{1}-\kappa_{2} u^{-\frac{1}{\alpha+2}}\left(X_{\bar{S}_{1}}-X_{0}\right) \cdot \ell\right\}\right]\right] .
\end{aligned}
$$

Since $\omega \in \mathcal{E}$, on the event $\left\{\bar{S}_{k}<T_{C_{4 L}}\right\}$, the inner expectation of the rightmost term is smaller than

$$
\exp \left\{\kappa_{1}+\kappa_{2} u^{-\frac{1}{\alpha+2}}\right\}\left(p_{0} e^{\kappa_{2}}+\left(1-p_{0}\right) e^{-\kappa_{2}}\right) \leq 1,
$$

provided we choose $\kappa_{2}>0$, small, make $\kappa_{1}$ sufficiently small and $u$ suitably large. Keeping such choices from now on, we see that the left member of (2.34) is smaller than:

$$
E_{x, \omega}\left[\exp \left\{\kappa_{1}(k \wedge V)-\kappa_{2} u^{-\frac{1}{\alpha+2}} X_{\bar{S}_{k \wedge V}} \cdot \ell\right\}, \bar{S}_{k \wedge V}<T_{C_{4 L}}\right],
$$

so that by induction it is smaller than:

$$
\exp \left\{-\kappa_{2} u^{-\frac{1}{\alpha+2}} x \cdot \ell\right\} .
$$

As a result letting $k$ tend to $\infty$, we see that for large $u$, arbitrary $\omega \in \mathcal{E}$, $x \in C_{4 L}$,

$$
E_{x, \omega}\left[\exp \left\{\kappa_{1} V\right\}, \bar{S}_{V}<T_{C_{4 L}}\right] \leq \exp \left\{2 \kappa_{2}\right\}
$$


Coming back to (2.30), (2.33), we see by induction that for large $u, \omega \in \mathcal{E}$, and $\lambda$ small enough so that $\psi(2 \lambda)<\kappa_{1}$,

$$
E_{0, \omega}\left[\exp \left\{\lambda u^{-\frac{2}{\alpha+2}} \bar{T}_{N}\right\}, \bar{T}_{N}<T_{C_{4 L}}\right] \leq \exp \left\{\kappa_{2} N\right\} .
$$

This and (2.29) proves (2.28), and concludes the proof of Theorem 2.4.

We can now apply the above theorem to walks which are neutral or point to the right, i.e. to the situation where $d \geq 1,(0.1)$ holds, and for suitable $v>0, \delta>0$ :

$$
\mathbb{P}\left[\omega(0, e)=\frac{1}{2 d}, \text { for all }|e|=1\right]=e^{-v},
$$

(we call neutral a site where $\omega(0, e)=\frac{1}{2 d}$, for all $|e|=1$ ),

$$
d(0, \omega) \cdot e_{1} \geq \delta, \mathbb{P} \text {-a.s., on the event }\{0 \text { is not neutral }\} .
$$

Apart from the additional assumption (0.1), this is precisely the setting of [24]. Choosing $\ell=e_{1}$, we see that (2.5) holds, moreover:

Theorem 2.5. Under the above assumptions,

$$
-\infty<\varliminf_{u \rightarrow \infty} u^{-\frac{d}{d+2}} \log P_{0}\left[\tau_{1}>u\right] \leq \varlimsup_{u \rightarrow \infty} u^{-\frac{d}{d+2}} \log P_{0}\left[\tau_{1}>u\right]<0 .
$$

Proof. The upper bound follows from Theorem 2.4 above and the fact that (2.15) holds with $\alpha=d$, as proven in Proposition 3.1 of [24]. As for the lower bound, introduce for integer $u \geq 1, U=\left[-\left[u^{\frac{1}{d+2}}\right],\left[u^{\frac{1}{d+2}}\right]\right]^{d}$, we have in view of the definition of $\tau_{1}$ :

$$
\begin{aligned}
& P_{0}\left[\tau_{1}>u\right] \geq P_{0}\left[X_{u} \cdot e_{1} \leq 0\right] \geq \\
& \mathbb{E}\left[\text { all sites of } U \text { are neutral, } P_{0, \omega}\left[T_{U}>u-d\left[u^{\frac{1}{d+2}}\right], X_{u} \cdot e_{1} \leq 0\right]\right] \geq \\
& \left.\exp \{-v|U|\} Q_{0}\left[T_{U}>u-d\left[u^{\frac{1}{d+2}}\right]\right](2 d)^{-d\left[u \frac{1}{d+2}\right.}\right],
\end{aligned}
$$

using the Markov property and selecting a nearest neighbor path in $U$ of length $d\left[u^{\frac{1}{d+2}}\right]$, joining $\left.X_{u-d[u} \frac{1}{d+2}\right]$ to some point with non-positive first component, and denoting by $Q_{0}$ the law of the simple random walk starting at the origin. It is classical that:

$$
\liminf _{u \rightarrow \infty} u^{-\frac{d}{d+2}} \log Q_{0}\left[T_{U}>u\right]>-\infty,
$$

and the lower bound in (2.39) follows. 
Remark 2.6. 1) Unlike what happens in the non-nestling case, cf. Theorem 2.1, there is no "universal tail behavior of $\tau_{1}$ " in the marginal nestling case.

To illustrate the point, consider the case where $\ell=e_{1}$, and observe that for $L>1$, when $\omega \in \Omega$ is such that $d(x, \omega) \cdot e_{1} \geq \frac{1}{L}$, for all $x \in \mathbb{Z}^{d}$, $\left(\rho^{X_{n} \cdot e_{1}}\right)_{n \geq 0}$ is a supermartingale under $P_{0, \omega}$, provided $\rho=\frac{1-1 / L}{1+1 / L}$. Using the stopping theorem, it then easily follows that:

$$
P_{0, \omega}\left[X_{T_{U_{L}}} \cdot e_{1} \leq-L\right] \leq \frac{1-\rho^{L}}{\rho^{-L}-\rho^{L}} \underset{L \rightarrow \infty}{\rightarrow} p=\frac{1-e^{-2}}{e^{2}-e^{-2}} \in\left(0, \frac{1}{2}\right) .
$$

If we define $p_{0}=\frac{1}{2}\left(\frac{1}{2}+p\right)<\frac{1}{2}$, and $D_{L}=\left\{x \in \mathbb{Z}^{d},\left|x \cdot e_{1}\right|<L\right.$, $\left.\left|x \cdot e_{i}\right| \leq L^{3}, i=2, \ldots, d\right\}$, with the help of (2.7), it is easy to deduce that for large $L$,

$$
\begin{aligned}
& \left\{\omega \in \Omega_{+}, P_{0, \omega}\left[X_{T_{U_{L}}} \cdot e_{1} \leq-L\right]>p_{0}\right\} \subseteq \\
& \left\{\omega \in \Omega_{+}, \text {for some } x \in D_{L}, d(x, \omega) \cdot e_{1}<\frac{1}{L}\right\} .
\end{aligned}
$$

But for arbitrary $c>1$, it is a simple matter to construct $\mu$ for which $(0.1)$ together with (2.5), relative to $\ell=e_{1}$, hold and

$$
\varlimsup_{s \rightarrow 0} s^{c} \log \mathbb{P}\left[d(0, \omega) \cdot e_{1} \leq s\right]<0 .
$$

This and (2.41) shows that in this case (2.15) holds with $\alpha=c$. As a result of Theorem 2.4, one sees that for arbitrary $c>0$, one has examples of marginal nestling walks for which

$$
\varlimsup_{u \rightarrow \infty} u^{-c} \log P_{0}\left[\tau_{1}>u\right]<0 .
$$

2) It is a simple matter to adapt the proof of Theorem 2.4 in the case where (2.15) is replaced by the assumption that

$$
\varlimsup_{L \rightarrow \infty} \varphi(L)^{-1} \log \mathbb{P}\left[P_{0, \omega}\left[X_{T_{U_{L}}} \cdot \ell \leq-L\right]>p_{0}\right]<0,
$$

where $\varphi(\cdot): \mathbb{R}_{+} \rightarrow \mathbb{R}_{+}$is nondecreasing and such that $\lim \frac{\varphi(L)}{L}=\infty$.

The conclusion (2.16) is then replaced by:

$$
\begin{aligned}
& \varlimsup_{u \rightarrow \infty} \Phi(u)^{-1} \log P_{0}\left[\tau_{1}>u\right]<0, \text { where } \\
& \Phi(u)=\sup _{L>0} \min \left(\varphi(L), \frac{u}{L^{2}}\right)(=o(u)) .
\end{aligned}
$$


The role of $u^{\frac{\alpha}{\alpha+2}}$ is taken by $\Phi(u)$, and that of $u^{\frac{1}{\alpha+2}}$ is taken by some function $L_{1}(u)$ such that $\varphi\left(L_{1}(u)\right) \wedge \frac{u}{L_{1}(u)^{2}}>\frac{1}{2} \Phi(u)$. The choice of $\varphi(\cdot)$ is easily seen to imply that $u^{\frac{1}{3}}=o(\Phi(u))$, and $L_{1}(u)=o\left(u^{\frac{1}{3}}\right)$.

We now turn to the

C) Plain nestling case

We assume now that 0 belongs to the interior of $K_{o}$ and $\ell \in S^{d-1}$ is such that (1.7) holds. Our main object in the remainder of this section is the proof of a lower bound on the tail of $\tau_{1}$ which highlights the difference of the present situation with cases $\mathrm{A}$ ) and $\mathrm{B}$ ).

Theorem 2.7. $(d \geq 1)$. Under (0.1) and the above,

$$
\varliminf_{u \rightarrow \infty}(\log u)^{-d} \log P_{0}\left[\tau_{1}>u\right]>-\infty .
$$

Proof. Since we are in the plain nestling case, a continuity argument shows that

$$
2 c_{11}(d, \mu) \stackrel{\text { def }}{=} \inf _{w \in S^{d-1}} \mathbb{E}\left[(d(0, \omega) \cdot w)_{-}\right]>0,
$$

and from (1.29),

$$
\mathbb{P}\left[(d(0, \omega) \cdot w)_{-} \geq c_{11}\right] \geq c_{11}^{2}, \text { for } w \in S_{d-1} .
$$

Define for $r>1$,

$$
B_{r}=\left\{x \in \mathbb{Z}^{d},|x| \leq r\right\} \text {, and }
$$

$$
\mathcal{E}_{r}=\left\{\omega: \forall x \in B_{r} \backslash\{0\}, d(x, \omega) \cdot \frac{x}{|x|} \leq-c_{11}\right\},
$$

as we shall see this event will define a "trap" on $B_{r}$ for the walk, cf. (2.54) below.

Lemma 2.8. There exists $c_{12}(d, \mu), c_{13}(d, \mu)>0$, such that when $r>c_{12}$ for $\omega \in \mathcal{E}_{r}$,

$$
E_{x, \omega}\left[f\left(X_{1}\right)\right] \leq f(x), \text { for } x \in B_{r} \backslash B_{c_{12}} \text {, with } f(x)=\exp \left\{c_{13}|x|\right\} .
$$

Proof. We pick $c>0, r>1$, and observe that for $x \neq 0$, and $\omega \in \Omega$,

(2.53) $E_{x, \omega}\left[\exp \left\{c\left|X_{1}\right|\right\}\right]=\exp \{c|x|\} \sum_{|e|=1} \omega(x, e) \exp \{c(|x+e|-|x|)\}$. 
However, if $\bar{x}=\frac{x}{|x|}$,

$$
\begin{aligned}
& \left|\sum_{|e|=1} \omega(x, e) \exp \{c(|x+e|-|x|)\}-1-c d(x, \omega) \cdot \bar{x}\right| \leq \\
& \sup _{|e|=1}\left|\exp \left\{c \frac{2 x \cdot e+1}{|x+e|+|x|}\right\}-1-c \frac{x}{|x|} \cdot e\right| \leq \\
& c \sup _{|e|=1}\left|\frac{2 x \cdot e+1}{|x+e|+|x|}-\frac{x}{|x|} \cdot e\right|+O\left(c^{2}\right)=c O\left(\frac{1}{|x|}\right)+O\left(c^{2}\right),
\end{aligned}
$$

$(|x|$ large, $c$ small).

Therefore, for $\omega \in \mathcal{E}_{r}, c_{12}<|x| \leq r$ and $c \leq c_{13}$,

$$
E_{x, \omega}\left[\exp \left\{c\left|X_{1}\right|\right\}\right] \leq \exp \{c|x|\},
$$

and the lemma follows.

The above lemma implies that when $r>c_{12}$, for $c_{12}<|x| \leq r, \omega \in \mathcal{E}_{r}$, $f\left(X_{n \wedge H_{B_{c_{12}}} \wedge T_{B_{r}}}\right)$ is a supermartingale under $P_{x, \omega}$ and it follows from the stopping theorem that:

$$
P_{x, \omega}\left[T_{B_{r}}<H_{B_{c_{12}}}\right] \leq \exp \left\{c_{13}(|x|-r)\right\} .
$$

Next observe that for large $u \in \mathbb{N}$, for a path starting in 0 , one way to reach 0 after time $u$ is to successively exit $B_{r} \backslash B_{c_{12}}$ through $B_{c_{12}}, u$ times, and then go to 0 . As a result, when $u$ is large, $\omega \in \mathcal{E}_{r}$, with $r=\frac{2}{c_{13}} \log u$,

$$
\begin{aligned}
P_{0, \omega}\left[\tau_{1}>u\right] & \geq P_{0, \omega}\left[H_{0} \circ \theta_{u}<\infty\right] \\
& \geq\left(\inf _{x \in \partial B_{c_{12}}} P_{x, \omega}\left[T_{B_{r}}>H_{B_{c_{12}}}\right]\right)^{u} \cdot \kappa^{\sqrt{d} c_{12}} \\
& \geq \kappa^{\sqrt{d} c_{12}}\left(1-\frac{1}{u}\right)^{u} \rightarrow \kappa^{\sqrt{d} c_{12}} e^{-1}, \text { as } u \rightarrow \infty .
\end{aligned}
$$

On the other hand

$$
\varliminf_{u \rightarrow \infty}(\log u)^{-d} \log \mathbb{P}\left[\mathcal{E}_{\frac{2}{c_{13}} \log u}\right]>-\infty,
$$

and our claim (2.47) follows from the above and (2.55).

Remark 2.9. If indeed "traps" govern the tail behavior of $\tau_{1}$, it is natural to expect in the present setting of C) that Theorem 2.7 in fact captures the true decay of the tail of $\tau_{1}$, in the sense that $\overline{\lim }(\log u)^{-d} \log P_{0}\left[\tau_{1}>u\right]$ $<0$. In the one-dimensional setting, such an upper bound easily follows from Lemma 1.3, and Theorem 1.1 of Dembo-Peres-Zeitouni [4]. The next section will present results in the direction of such an upper bound in the higher dimensional setting. 


\section{A priori tail estimates for the renewal times}

Throughout this section we assume that the dimension $d \geq 2$, and (0.1) together with (1.7) hold. The main objective here is to derive an upper bound on the tail of $\tau_{1}$ under $P_{0}$, cf. Theorem 3.5. In particular for plain nestling walks, this partly complements the lower estimates of Theorem 2.7.

We begin with a proposition which presents an estimate in the spirit of (2.15). Recall the definition of $U_{L}$ in (2.8).

Proposition 3.1. $(d \geq 2)$. For $\beta \in[0,1]$ and $c>0$,

$$
\begin{gathered}
\varlimsup_{L \rightarrow \infty} L^{-\zeta} \log \mathbb{P}\left[P_{0, \omega}\left[X_{T_{U_{L}}} \cdot \ell \geq L\right] \leq e^{-c L^{\beta}}\right]<0, \text { where } \\
\zeta=d \text {, if } \beta=1 \text {, and either } \zeta=1 \text {, or } \zeta<d(3 \beta-2) \text {, if } \beta<1 .
\end{gathered}
$$

Proof. We begin with the simple observation that (3.1) holds with $\zeta=1$, regardless of the value of $\beta \in[0,1]$ and $c>0$. Indeed with the notations of (0.5) and (1.40),

$$
\begin{aligned}
& \varlimsup L^{-1} \log \mathbb{P}\left[P_{0, \omega}\left[X_{T_{U_{L}}} \cdot \ell \geq L\right] \leq e^{-c}\right]= \\
& \varlimsup L^{-1} \log \mathbb{P}\left[P_{0, \omega}\left[\widetilde{T}_{-L}<T_{L}\right] \geq 1-e^{-c}\right] \leq \\
& \varlimsup L^{-1} \log \left(\left(1-e^{-c}\right)^{-1} P_{0}\left[\widetilde{T}_{-L}<T_{L}\right]\right)<0,
\end{aligned}
$$

using a similar estimate as in (1.44).

As a result we need only consider from now on $\beta \in[0,1]$, large enough so that:

$$
d(3 \beta-2)>1 .
$$

The idea of the proof is to construct, under appropriate requirements on the environment, strategies for the walk, which ensure that it exits $U_{L}$ through the part $\partial_{+} U_{L}$ of its boundary where $\{x \cdot \ell \geq L\}$. We then prove that it is unlikely that the environment does not fulfill these requirements. The construction of these strategies will involve suitable notions of "good boxes" and "bad boxes".

We now turn to the definition of good and bad blocks. We first need some further notations. We choose a rotation $\widetilde{R}$ of $\mathbb{R}^{d}$, such that

$$
\widetilde{R}\left(e_{1}\right)=\frac{v}{|v|} .
$$


We consider $\gamma \in\left(\frac{1}{2}, 1\right)$, and $L_{0}$ such that $L_{0}^{\gamma} \geq 2 \sqrt{d}$, and define for $z \in L_{0} \mathbb{Z}^{d}$, the $z$-blocks:

$$
\begin{aligned}
& \widetilde{B}_{1}(z)=\widetilde{R}\left(z+\left[0, L_{0}\right]^{d}\right) \cap \mathbb{Z}^{d}\left(\neq \emptyset, \text { since } L_{0} \geq \sqrt{d}\right) \\
& \widetilde{B}_{2}(z)=\widetilde{R}\left(z+\left(-L_{0}^{\gamma}, L_{0}+L_{0}^{\gamma}\right)^{d}\right) \cap \mathbb{Z}^{d},
\end{aligned}
$$

(let us stress that $\left[0, L_{0}\right]^{d}$ and $\left(-L_{0}^{\gamma}, L_{0}+L_{0}^{\gamma}\right)^{d}$ denote solid blocks of $\mathbb{R}^{d}$, and not their restriction to $\mathbb{Z}^{d}$ ). We also define the "top boundary" of $\widetilde{B}_{2}(z)$ :

$$
\partial_{+} \widetilde{B}_{2}(z)=\partial \widetilde{B}_{2}(z) \cap\left\{x: x \cdot \widetilde{R}\left(e_{1}\right) \geq L_{0}+L_{0}^{\gamma}\right\} .
$$

We shall say that $z \in L_{0} \mathbb{Z}^{d}$ is $L_{0}$-good, when

$$
\sup _{x \in \widetilde{B}_{1}(z)} P_{x, \omega}\left[X_{\widetilde{B}_{2}(z)} \notin \partial_{+} \widetilde{B}_{2}(z)\right] \leq \frac{1}{2},
$$

and $L_{0}$-bad otherwise. We then have the following control on the probability that a box is $L_{0}$-bad:

Lemma 3.2. If $\gamma \in\left(\frac{1}{2}, 1\right)$, then

$$
\varlimsup_{L_{0} \rightarrow \infty} L_{0}^{1-2 \gamma} \sup _{z \in L_{0} \mathbb{Z}^{d}} \log \mathbb{P}\left[z \text { is } L_{0}-b a d\right]<0 .
$$

Proof. For $z \in L_{0} \mathbb{Z}^{d}$

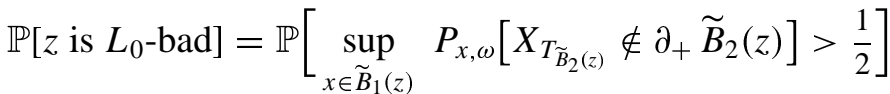

$$
\begin{aligned}
& \leq 2\left|\widetilde{B}_{1}(z)\right| \sup _{x \in \widetilde{B}_{1}(z)} P_{x}\left[X_{T_{\widetilde{B}_{2}(z)}} \notin \partial_{+} \widetilde{B}_{2}(z)\right] .
\end{aligned}
$$

Observe that for $x \in \widetilde{B}_{1}(z), \widetilde{B}_{2}(z)$ is included in the closed Euclidean ball centered at $x$ of radius $3 \sqrt{d} L_{0}$, so that

$$
P_{x} \text {-a.s., } T_{\widetilde{B}_{2}(z)} \leq T_{x \cdot \ell+3 \sqrt{d} L_{0}} .
$$

Further, $P_{x}$-a.s. on the event $\left\{X_{\widetilde{B}_{\widetilde{B}_{2}(z)}} \notin \partial_{+} \widetilde{B}_{2}(z)\right\}$,

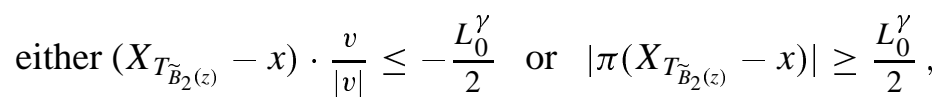

where $\pi(\cdot)$ in the notations of Corollary 1.5 stands for the orthogonal projection on the orthogonal complement of $v$. We thus see that 


$$
\begin{aligned}
& \mathbb{P}\left[z \text { is } L_{0} \text {-bad }\right] \leq c(d) L_{0}^{d}\left(P_{0}\left[\sup _{0 \leq n \leq T_{3 \sqrt{d} L_{0}}}\left|\pi\left(X_{n}\right)\right| \geq \frac{v \cdot \ell}{4} L_{0}^{\gamma}\right]\right. \\
& \left.+P_{0}\left[\sup _{0 \leq n \leq T_{3 \sqrt{d} L_{0}}}\left|\pi\left(X_{n}\right)\right|<\frac{v \cdot \ell}{4} L_{0}^{\gamma}, \inf _{0 \leq n \leq T_{3 \sqrt{d} L_{0}}} X_{n} \cdot \frac{v}{|v|} \leq-\frac{L_{0}^{\gamma}}{2}\right]\right) \\
& \leq c(d) L_{0}^{d}\left(P_{0}\left[\sup _{0 \leq n \leq T_{3 \sqrt{d} L_{0}}}\left|\pi\left(X_{n}\right)\right| \geq \frac{v \cdot \ell}{4} L_{0}^{\gamma}\right]+P_{0}\left[\widetilde{T}_{-\frac{L_{0}^{\gamma}}{4} v \cdot \ell}<\infty\right]\right),
\end{aligned}
$$

where we used the fact that $X_{n} \cdot \ell \leq\left(X_{n} \cdot \frac{v}{|v|}\right)\left(\frac{v \cdot \ell}{|v|}\right)+\left|\pi\left(X_{n}\right)\right|$, and $|v| \leq 1$, to obtain the rightmost term of the last line in (3.10). The claim now follows from (1.56) and (1.60).

We shall now pile up boxes in the direction $v$, to form columns and then gather columns to form tubes, more precisely, for $L>0$ (as in (3.2)), and $L_{0}$ as above (the relation of $L_{0}$ to $L$ appears in (3.21) below), we attach to each $z \in L_{0} \mathbb{Z}^{d}$, the column

$$
\operatorname{Col}(z)=\left\{z^{\prime} \in L_{0} \mathbb{Z}^{d}, \exists j \in[0, J], z^{\prime}=z+j L_{0} e_{1}\right\}, \text { where }
$$

$J$ is the smallest integer such that $J L_{0} \frac{v}{|v|} \cdot \ell \geq 3 L$.

Choosing $L_{1}>0$, some integer multiple of $L_{0}$, we define the tube attached to $z \in L_{0} \mathbb{Z}^{d}$ :

$$
\begin{aligned}
& \operatorname{Tube}(z)= \\
& \left\{z^{\prime} \in L_{0} \mathbb{Z}^{d}, \exists j_{2}, \ldots, j_{d} \in\left[0, \frac{L_{1}}{L_{0}}-1\right], z^{\prime}=z+\sum_{i=2}^{d} j_{i} L_{0} e_{i}\right\},
\end{aligned}
$$

(recall $\left(e_{i}\right)$ denotes the canonical basis of $\mathbb{R}^{d}$, see above (1.45)).

The rough idea behind these definitions, is that one way for the walk to exit $U_{L}$ is to move to one of the "bottom blocks" in Tube $(0)$ of an appropriate column containing many $L_{0}$-good blocks, and essentially move along this column up to its top. With the choices of $L_{0}, L_{1}$ we later make, cf. (3.27), this will indeed ensure exiting $U_{L}$ through $\partial_{+} U_{L}\left(=\partial U_{L} \cap\{x: x \cdot \ell \geq L\}\right)$. We now define the top of a tube as:

$$
\operatorname{top}(z)=\bigcup_{z^{\prime} \in \operatorname{Tube}(z)} \partial_{+} \widetilde{B}_{2}\left(z^{\prime}+J L_{0} e_{1}\right), z \in L_{0} \mathbb{Z}^{d},
$$

as well as the neighborhood of a tube:

$$
V(z)=\left\{x \in \mathbb{Z}^{d}, \exists y \in \bigcup_{\substack{z^{\prime} \in \text { Tube }(z), 0 \leq j \leq J}} \widetilde{B}_{1}\left(z^{\prime}+j L_{0} e_{1}\right),\|x-y\| \leq 3 d L_{1}\right\}
$$


To derive a lower bound on the $P_{0, \omega}$-probability of reaching the top of the tube attached to 0 , before exiting its neighborhood, it is convenient to introduce the minimum number of $L_{0}$-bad boxes contained in a column among columns within a tube:

$$
n(z, \omega)=\min _{z^{\prime} \in \operatorname{Tube}(z)}\left(\sum_{j=0}^{J} 1\left\{z^{\prime}+L_{0} j e_{1} \text { is } L_{0} \text {-bad }\right\}\right) .
$$

Lemma 3.3. There exists $c_{14}(d)$, such that for any tube and any site within a tube, i.e. for any $z \in L_{0} \mathbb{Z}^{d}$ and any

$$
\begin{aligned}
& x \in \bigcup_{z^{\prime} \in \text { Tube }(z), 0 \leq j \leq J} \widetilde{B}_{1}\left(z^{\prime}+j L_{0} e_{1}\right) \stackrel{\text { def }}{=} D(z), \\
& P_{x, \omega}\left[H_{\mathrm{top}(z)}<T_{V(z)}\right] \geq \kappa^{c_{14}\left(L_{1}+J L_{0}^{\gamma}+n(z, \omega) L_{0}\right)}\left(\frac{1}{2}\right)^{J+1} .
\end{aligned}
$$

Proof. In view of (1.1), any point in $D(z)$ can be joined by a nearest neighbor path of length at most $d L_{1}$ to some $\bar{x}$ in some $\widetilde{B}_{1}\left(\bar{z}+\bar{j} L_{0} e_{1}\right), \bar{j} \in[0, J]$ and $\bar{z} \in \operatorname{Tube}(z)$ such that:

$$
n(z, \omega)=\sum_{j=0}^{J} 1\left\{\bar{z}+j L_{0} e_{1} \text { is } L_{0} \text {-bad }\right\} .
$$

Observe also that any point of $\partial_{+} \widetilde{B}_{2}\left(\bar{z}+\bar{j} L_{0} e_{1}\right)$ is within $|\cdot|$-distance at most $3 \sqrt{d} L_{0}^{\gamma}$ from some point in $\widetilde{B}_{1}\left(\bar{z}+(\bar{j}+1) L_{0} e_{1}\right)$, and can thus be joined by a nearest neighbor path to this point with length at most $3 d L_{0}^{\gamma}$. Thus, if $\bar{z}+\bar{j} L_{0} e_{1}$ is $L_{0}$-good, using the strong Markov property, (3.8) and (0.1):

$$
P_{\bar{x}, \omega}\left[H_{\widetilde{B}_{1}\left(\bar{z}+(\bar{j}+1) L_{0} e_{1}\right)}<T_{V(z)}\right] \geq \frac{1}{2} \kappa^{3 d L_{0}^{\gamma}} .
$$

On the other hand, if $\bar{z}+\bar{j} L_{0} e_{1}$ is $L_{0}$-bad, $\bar{x}$ is within $|\cdot|$-distance $L_{0}+d$ of some point in $B_{1}\left(\bar{z}+(\bar{j}+1) L_{0} e_{1}\right)$, and by a similar argument:

$$
P_{\bar{x}, \omega}\left[H_{\widetilde{B}_{1}\left(\bar{z}+(\bar{j}+1) L_{0} e_{1}\right)}<T_{V(z)}\right] \geq \kappa^{2 d L_{0}} .
$$

Using the strong Markov property repeatedly and estimates as (3.17), (3.18), we obtain (3.16).

In view of Lemma 3.2, we now choose $\gamma$ with $1>\gamma>\frac{1}{2}$ such that:

$$
\chi \stackrel{\text { def }}{=} \frac{1-\beta}{1-\gamma}<\beta \leq 1,
$$

such a choice indeed possible thanks to (3.4). We then choose

$$
v>1-\gamma,
$$


and introduce for large $L$ :

$$
L_{0}=\rho_{1} L^{\chi}, L_{1}=\left[\rho_{2} L^{\beta-\chi}\right] L_{0}, N_{0}=\left[\rho_{3} L^{\beta-\chi}\right],
$$

where the constants $\rho_{i}, i=1,2,3$, possibly depend on $d, c$ in (3.1), $v, \ell, \kappa, \epsilon$, and are selected so that for large $L$ :

$$
\begin{gathered}
\kappa^{c_{14} J L_{0}^{\gamma}}, \kappa^{c_{14} L_{1}}, \kappa^{c_{14} N_{0} L_{0}},\left(\frac{1}{2}\right)^{J+1}>\exp \left\{-\frac{c}{5} L^{\beta}\right\}, \\
\frac{N_{0}}{3}>(J+1) \frac{\left(e^{2}-1\right)}{L_{0}^{v}}, \text { and }
\end{gathered}
$$

any nearest neighbor path within $V(0)$, between 0 and Top $(0)$,

first exits $U_{L}$ through $\partial_{+} U_{L}$.

To see that such a choice is possible observe that it suffices to choose $\rho_{1}$ large enough and $\rho_{2}=\rho_{3}=c\left(10 \rho_{1} c_{14} \log \frac{1}{\kappa}\right)^{-1}$, then (3.22), (3.24) hold for large $L$. As for (3.23), when $\beta<1$, it follows from the inequality $\beta-\chi>1-(1+v) \chi$, on the other hand when $\beta=1$, (3.23) is also seen to hold if $\rho_{1}$ is large.

Note that the events $\left\{z\right.$ is $L_{0}$-good $\}$, where $z$ runs over the collection of $\left(k_{1} L_{0}, \ldots, k_{d} L_{0}\right)$, where each $k_{1}, \ldots, k_{d}$ has a fixed parity, are jointly independent. Therefore:

$$
\begin{aligned}
& \mathbb{P}\left[n(0, \omega)>N_{0}\right] \leq \\
& \quad\left(\sup _{z^{\prime} \in \text { Tube }(0)} \mathbb{P}\left[\sum_{0}^{J} 1\left\{z^{\prime}+j L_{0} e_{1} \text { is } L_{0}-\text { bad }\right\}>N_{0}\right]\right)^{\left[\frac{L_{1}}{2 L_{0}}\right]^{d-1}} .
\end{aligned}
$$

Observe that when $Z$ is a Bernoulli variable with success probability smaller than $L_{0}^{-v}, E[\exp \{2 Z\}] \leq 1+\left(e^{2}-1\right) L_{0}^{-\nu}$. Thus restricting $j$ to even or odd integers, we conclude from Chebyshev inequality with the help of Lemma 3.2, choosing $\rho_{1}$ sufficiently large when $\beta=1$, and the choice of $v$ in (3.20), that for large $L$

$$
\begin{aligned}
\sup _{z^{\prime}} \mathbb{P}[ & \left.\sum_{0}^{J} 1\left\{z^{\prime}+j L_{0} e_{1} \text { is } L_{0} \text {-bad }\right\}>N_{0}\right] \\
& \leq 2 \exp \left\{-N_{0}\right\}\left(1+\frac{e^{2}-1}{L_{0}^{\nu}}\right)^{J+1} \\
& \leq 2 \exp \left\{-N_{0}+(J+1) \frac{\left(e^{2}-1\right)}{L_{0}^{\nu}}\right\} \stackrel{(3.23)}{\leq} \exp \left\{-\frac{N_{0}}{2}\right\} .
\end{aligned}
$$

Thus for large $L$,

$$
\mathbb{P}\left[n(0, \omega)>N_{0}\right] \leq \exp \left\{-\frac{N_{0}}{2}\left[\frac{L_{1}}{2 L_{0}}\right]^{d-1}\right\},
$$


whereas on the event $\left\{n(0, \omega) \leq N_{0}\right\}$ :

$$
P_{0, \omega}\left[X_{T_{U_{L}}} \cdot \ell \geq L\right] \stackrel{(3.24)}{\geq} P_{0, \omega}\left[H_{\mathrm{Top}(0)}<T_{V(0)}\right] \stackrel{(3.16)-(3.22)}{>} e^{-c L^{\beta}} .
$$

Therefore

$$
\varlimsup_{L \rightarrow \infty} L^{-d(\beta-\chi)} \log \mathbb{P}\left[P_{0, \omega}\left[X_{T_{U_{L}}} \cdot \ell \geq L\right] \leq e^{-c L^{\beta}}\right]<0 .
$$

Letting $\gamma$ vary according to (3.19) completes the proof of Proposition 3.1. Let us add a comment. It is possible to take (superficially) fuller advantage of the estimate (3.9), as can be suspected from the fact that $v$ in (3.20) can be chosen arbitrarily large. This leads to a term $(2 \gamma-1) \chi+d(\beta-\chi)$ in place of $d(\beta-\chi)$ in the last inequality above. Optimizing over $\gamma$, one still chooses $\gamma$ close to $\frac{1}{2}$, so that no additional advantage results from this fuller use of Lemma 3.2.

Remark 3.4. Let us mention that in the plain nestling case (under $(0.1)$ and (1.7)), the estimate (3.1), when $\beta=1$, and $c$ is small, is reasonably sharp. Indeed one can use a "trap" of the type described in (2.51), with $r$ of the order $\frac{3}{2} L$ and center close to $-\left(c_{12}+L\right) \ell$, to see with the help of (2.54), that for small $c$ :

$$
\varliminf_{L \rightarrow \infty} L^{-d} \log \mathbb{P}\left[P_{0, \omega}\left[X_{T_{U_{L}}} \cdot \ell \geq L\right] \leq e^{-c L}\right]>-\infty .
$$

Of course for large $c$, the probability under the logarithm equals 0 , due to assumption (0.1), and the estimate (3.1) with $\beta=1$, cannot be sharp in this case.

We now come to the main result of this section, namely

Theorem 3.5. $(d \geq 2)$. Assume (0.1) and (1.7), then for $\alpha<1+\frac{d-1}{3 d}$,

$$
\varlimsup_{u \rightarrow \infty}(\log u)^{-\alpha} \log P_{0}\left[\tau_{1}>u\right]<0,
$$

(in particular $\tau_{1}$ has finite $P_{0}$-moments of arbitrary order).

Proof. Choose $\alpha \in\left(1,1+\frac{d-1}{3 d}\right)$ and define for large $u$

$$
\begin{array}{r}
\Delta(u)=\frac{1}{10 \sqrt{d}} \frac{\log u}{\log \frac{1}{\kappa}} \text { and } L(u)=N(u) \Delta(u), \\
\text { where } N(u)=\left[(\log u)^{\alpha-1}\right] .
\end{array}
$$

For simplicity we shall drop $u$ from the notation in what follows. In view of Lemma 1.3, (3.29) will follow from 


$$
\varlimsup_{u \infty}(\log u)^{-\alpha} \log P_{0}\left[T_{C_{L}}>u\right]<0 .
$$

Note that for large $u$,

$$
\begin{aligned}
& P_{0}\left[T_{C_{L}}>u\right] \leq \\
& \mathbb{E}\left[\text { for all } x \in C_{L}, P_{x, \omega}\left[T_{C_{L}} \leq \frac{u}{(\log u)^{\alpha}}\right] \geq \frac{1}{2}, P_{0, \omega}\left[T_{C_{L}}>u\right]\right] \\
& \quad+\mathbb{P}\left[\text { for some } x_{1} \in C_{L}, P_{x_{1}, \omega}\left[T_{C_{L}}>\frac{u}{(\log u)^{\alpha}}\right] \geq \frac{1}{2}\right] .
\end{aligned}
$$

Using the simple Markov property, the first term in the right hand side of (3.32) is smaller than:

$$
\left(\frac{1}{2}\right)^{\left[(\log u)^{\alpha}\right]}
$$

As for the second term in the right hand side of (3.32), notice that when $P_{x_{1}, \omega}\left[T_{C_{L}}>\frac{u}{(\log u)^{\alpha}}\right] \geq \frac{1}{2}$,

$$
\frac{1}{2} \frac{u}{(\log u)^{\alpha}} \leq E_{x_{1}, \omega}\left[T_{C_{L}}\right]=\sum_{x \in C_{L}} \frac{P_{x_{1}, \omega}\left[H_{x}<T_{C_{L}}\right]}{P_{x, \omega}\left[\widetilde{H}_{x}>T_{C_{L}}\right]},
$$

using in the last step a standard Markov chain calculation and denoting by $\widetilde{H}(x)$ the hitting time of $\{x\}$ :

$$
\widetilde{H}_{x}=\inf \left\{n \geq 1, X_{n}=x\right\} .
$$

As a result for some $x_{2} \in C_{L}$,

$$
P_{x_{2}, \omega}\left[\widetilde{H}_{x_{2}}>T_{C_{L}}\right] \leq \frac{2(\log u)^{\alpha}}{u}\left|C_{L}\right| .
$$

Observe also that when $u$ is sufficiently large, for arbitrary $\omega \in \Omega, y \in C_{L}$, $x \in \mathbb{Z}^{d}$ with $\|y-x\| \leq \frac{1}{3} \frac{\log u}{\log \frac{1}{\kappa}}$, (in particular when $(|y-x| \leq 2 \Delta+d)$ ),

$$
P_{y, \omega}\left[\widetilde{H}_{y}>T_{C_{L}}\right] \stackrel{(0.1)}{\geq} u^{-1 / 3} P_{x, \omega}\left[H_{y}>T_{C_{L}}\right] .
$$

Introduce now the notation

$$
\mathcal{G}_{i}=\partial\left\{z \in \mathbb{Z}^{d}, z \cdot \ell<i \Delta\right\}, \text { for } i \in \mathbb{Z},
$$

it follows from (3.36) and (3.37), (with $x_{2}$ in the place of $y$ ), that for large $u$ on the event:

$$
\mathcal{R} \stackrel{\text { def }}{=} \bigcup_{x_{1} \in C_{L}}\left\{\omega: P_{x_{1}, \omega}\left[T_{C_{L}}>\frac{u}{(\log u)^{\alpha}}\right] \geq \frac{1}{2}\right\},
$$

we can find $i_{0} \in[-N+2, N-1]$ and $x_{0} \in C_{L} \cap \mathcal{G}_{i_{0}}$, so that

$$
P_{x_{0}, \omega}\left[\widetilde{T}_{\left(i_{0}-1\right) \Delta}>T_{C_{L}}\right] \leq \frac{1}{\sqrt{u}} \text {, with the notation of (1.40)) . }
$$


We now introduce for $i \in \mathbb{Z}$,

$$
\begin{aligned}
X_{i} & =-\log \inf _{x \in C_{L} \cap \mathcal{G}_{i}} P_{x, \omega}\left[\widetilde{T}_{(i-1) \Delta}>T_{(i+1) \Delta}\right], & \text { if } & C_{L} \cap \mathcal{G}_{i} \neq \emptyset \\
& =0 & \text {, if } & C_{L} \cap \mathcal{G}_{i}=\emptyset .
\end{aligned}
$$

The next inequality is obvious when $i=N$, and follows by induction and the strong Markov property for $i \in[-N+1, N], x \in \mathcal{G}_{i}$ :

$$
P_{x, \omega}\left[\widetilde{T}_{(i-1) \Delta}>T_{C_{L}}\right] \geq \exp \left\{-\sum_{j=i}^{N} X_{j}\right\} .
$$

This and (3.39) shows that for large $u$ :

$$
\mathbb{P}[\mathcal{R}] \leq \mathbb{P}\left[\sum_{-N+1}^{N} X_{i} \geq \frac{\log u}{2}\right] \leq 2 N \sup _{[-N+1, N]} \mathbb{P}\left[X_{i} \geq \frac{\log u}{2 N}\right] .
$$

Moreover for $v>0$ and $i \in \mathbb{Z}$, in the notation of (3.1) or (2.8),

$$
\mathbb{P}\left[X_{i}>v\right] \leq\left|C_{L}\right| \mathbb{P}\left[P_{0, \omega}\left[X_{T_{U_{\Delta}}} \cdot \ell \geq \Delta\right] \leq e^{-v}\right] .
$$

Thus for large $u$ :

$$
P_{0}\left[T_{C_{L}}>u\right] \leq\left(\frac{1}{2}\right)^{\left[(\log u)^{\alpha}\right]}+\mathbb{P}[\mathcal{R}],
$$

and as a result of (3.42), for large $u$ :

$$
P_{0}\left[T_{C_{L}}>u\right] \leq\left(\frac{1}{2}\right)^{\left[(\log u)^{\alpha}\right]}+\mathbb{P}\left[\Sigma_{e} X_{i} \geq \frac{\log u}{4}\right]+\mathbb{P}\left[\Sigma_{o} X_{i} \geq \frac{\log u}{4}\right]
$$

where $\Sigma_{e}$ and $\Sigma_{o}$ respectively denote the sum over even and odd $i$ in $[-N+1, N]$. The variables $X_{i}, i$ even, are independent, and the same holds for the variables $X_{i}, i$ odd. Thus for $\delta>0$, and large $u$, with hopefully obvious notations:

$$
\begin{aligned}
& \mathbb{P}\left[\Sigma_{e} X_{i} \geq \frac{\log u}{4}\right] \\
& \leq \exp \left\{-\frac{1}{4}(\log u)^{1+\delta}\right\} \prod_{e} E_{0}\left[\exp \left\{(\log u)^{\delta} X_{i}\right\}\right], \\
& \text { and } \sup _{i \in \mathbb{Z}} E_{0}\left[\exp \left\{(\log u)^{\delta} X_{i}\right\}\right] \\
& \stackrel{(3.43)}{\leq} 1+\int_{0}^{\infty}(\log u)^{\delta} e^{(\log u)^{\delta} v}\left|C_{L}\right| \mathbb{P}\left[P_{0, \omega}\left[\widetilde{T}_{-\Delta}>T_{\Delta}\right] \leq e^{-v}\right] d v,
\end{aligned}
$$

where the above integral is in fact concentrated, as a result of (0.1), on the interval $\left[0, c_{15}(d, \kappa) \Delta\right]$. If we introduce for $M \geq 1$,

$$
\beta_{j}=\frac{j}{M}, \quad 0 \leq j \leq M,
$$


we see by breaking the above integral over the intervals $\left[\left(c_{15} \Delta\right)^{\beta_{j}}\right.$, $\left.\left(c_{15} \Delta\right)^{\beta_{j+1}}\right], 0 \leq j<M$, that for suitable $c_{16}(d, \kappa), c_{17}(d, \kappa)>0, \delta>0$, and large $u$ :

$$
\begin{gathered}
\sup _{i \in \mathbb{Z}} E_{0}\left[\exp \left\{(\log u)^{\delta} X_{i}\right\}\right] \leq \\
1+c_{15} \Delta(\log u)^{\delta}\left|C_{L}\right| \sum_{j=0}^{M-1} \exp \left\{c_{16}(\log u)^{\delta+\beta_{j}+\frac{1}{M}}\right\} \\
\mathbb{P}\left[P_{0, \omega}\left[\widetilde{T}_{-\Delta}>T_{\Delta}\right] \leq e^{-c_{17}(\log u)^{\beta_{j}}}\right]
\end{gathered}
$$

Using (3.1), we see that:

$$
\begin{aligned}
& \varlimsup_{u \rightarrow \infty}(\log u)^{-\rho} \log \sup _{i \in \mathbb{Z}} E_{0}\left[\exp \left\{(\log u)^{\delta} X_{i}\right\}\right] \leq 0, \text { if } \\
& \rho>\sup _{0 \leq j \leq M}\left\{\delta+\beta_{j}+\frac{1}{M} ; \delta+\beta_{j}+\frac{1}{M} \geq \max \left(1, d\left(3 \beta_{j}-2\right)\right)\right\},
\end{aligned}
$$

(this supremum is understood as 0 if the set is empty).

Letting $M$ tend to infinity, we see that (3.48) holds if

$$
\begin{aligned}
\rho>\rho_{\delta} \stackrel{\text { def }}{=} & \sup \{\delta+\beta ; 0 \leq \beta \leq 1, \delta+\beta \geq \max (1, d(3 \beta-2))\} \\
& \quad \text { (with the convention } \rho_{\delta}=0, \text { if the above set is empty). }
\end{aligned}
$$

Coming back to (3.46), we find

$$
\begin{aligned}
\varlimsup_{u \rightarrow \infty}(\log u)^{-(1+\delta)} \log \mathbb{P}\left[\Sigma_{e} X_{i} \geq \frac{\log u}{4}\right]<0, \\
\text { when } 1+\delta>\alpha-1+\rho_{\delta},
\end{aligned}
$$

and a similar inequality holds with $\Sigma_{o} X_{i}$ in place of $\Sigma_{e} X_{i}$. Choosing $\delta=\alpha-1<\frac{d-1}{3 d}$, we find $\rho_{\delta}=0$, and thus $1+\delta>\alpha-1+\rho_{\delta}$, so that our claim (3.29) follows from (3.45), (3.50) and its analogue for $\Sigma_{o} X_{i}$.

Remark 3.6. 1) If one only uses the rightmost expression of (3.42) to bound $\mathbb{P}[\mathcal{R}]$, one obtains a weaker bound in (3.29), where $\alpha$ is restricted to be smaller than $\frac{4 d}{3 d+1}$ (which is smaller than $1+\frac{d-1}{3 d}=\frac{4 d-1}{3 d}$, when $d \geq 2$ ).

2) Observe that in the one-dimensional case, in the plain nestling situation, i.e. when $\mathbb{P}[\omega(0,1)>\omega(0,-1)]$ and $\mathbb{P}[\omega(0,1)<\omega(0,-1)]$ are both positive, $\tau_{1}$ always has some divergent moment, cf. Theorem 2.7.

3) It is natural to wonder whether (3.29) matter of factly holds with $\alpha=d$ $(\geq 2)$ and matches the lowerbound of Theorem 2.7. 


\section{Central limit theorem}

In this section we shall develop some applications of the tail estimates of the previous sections, to the derivation of a functional central limit theorem for the process

$$
B_{t}^{n}=\frac{1}{\sqrt{n}}\left(X_{[t n]}-[t n] v\right), t \geq 0,
$$

introduced in (0.8). We denote by $D\left(\mathbb{R}_{+}, \mathbb{R}^{d}\right)$ the set of $\mathbb{R}^{d}$-valued functions on $\mathbb{R}_{+}$, which are right-continuous and possess left limits. We endow this set with the Skorohod topology and its Borel $\sigma$-field, so that $B^{n}$. defines a $D\left(\mathbb{R}_{+}, \mathbb{R}^{d}\right)$-valued random variable. It will be convenient in this section to restrict the parameter $a>0$, entering the definition of $\tau_{1}$, see after (1.13), to values large enough so that the strip $\left\{x \in \mathbb{Z}^{d}, 0 \leq x \cdot \ell<a\right\}$, is connected. In view of the discussion below (1.25), it suffices to choose $a>2 \sqrt{d}$, and keeping in mind (1.14), we assume:

$$
2 \sqrt{d}<a \leq 10 \sqrt{d} .
$$

Theorem 4.1 ( $d \geq 1)$. Assume (0.1), (1.7), (4.2) and

$$
E_{0}\left[\tau_{1}^{2} \mid D=\infty\right]<\infty
$$

then $B^{n}$. converges in law under $P_{0}$ to a non-degenerate d-dimensional Brownian motion with covariance matrix

$$
A=E_{0}\left[\left(X_{\tau_{1}}-\tau_{1} v\right)^{t}\left(X_{\tau_{1}}-\tau_{1} v\right) \mid D=\infty\right] / E_{0}\left[\tau_{1} \mid D=\infty\right] .
$$

Proof. Define the non-decreasing sequence $k_{n}, n \geq 0, P_{0}$-a.s. tending to $+\infty$, such that: $\tau_{k_{n}} \leq n<\tau_{k_{n}+1}$, with the convention $\tau_{0}=0$.

It follows from the strong law of large numbers and (1.19), that

$$
P_{0} \text {-a.s., } \frac{k_{n}}{n} \longrightarrow \frac{1}{E_{0}\left[\tau_{1} \mid D=\infty\right]}, \text { as } n \rightarrow \infty,
$$

and using Dini's theorem:

$$
P_{0} \text {-a.s., for all } T>0, \sup _{0 \leq t \leq T}\left|\frac{k_{[t n]}}{n}-\frac{t}{E_{0}\left[\tau_{1} \mid D=\infty\right]}\right| \underset{n \rightarrow \infty}{\longrightarrow} 0 .
$$

The random variables

$$
Z_{j}=X_{\tau_{j+1}}-X_{\tau_{j}}-\left(\tau_{j+1}-\tau_{j}\right) v, j \geq 1,
$$


in view of (1.19) and the definition of $v$ in (1.21), are i.i.d., centered under $P_{0}$, and thanks to (4.3), square integrable. Let us denote by $\Sigma_{m}, m \geq 0$, the partial sums:

$$
\Sigma_{m}=\sum_{j \leq m} Z_{j}, m \geq 0 .
$$

Observe that $P_{0}$-a.s., for any $T>0$ :

$$
\sup _{t \leq T}\left|B_{t}^{n}-\frac{\Sigma_{k_{[t n]}}}{\sqrt{n}}\right| \leq 2(1+|v|) \sup _{0 \leq k \leq k_{[T n]}} \frac{\tau_{k+1}-\tau_{k}}{\sqrt{n}} .
$$

Notice also that:

$$
\sup _{0 \leq k \leq k_{[n]}} \frac{\tau_{k+1}-\tau_{k}}{\sqrt{n}} \underset{n \rightarrow \infty}{\longrightarrow} 0, \text { in } P_{0} \text {-probability, }
$$

since in view of (1.19), and the inequality $k_{n} \leq n$, for $u>0$ :

$$
\begin{aligned}
& P_{0}\left[\sup _{0 \leq k \leq k_{[T n]}} \frac{\tau_{k+1}-\tau_{k}}{\sqrt{n}}>u\right] \\
& \leq P_{0}\left[\tau_{1}>\sqrt{n} u\right]+(n T+1) P_{0}\left[\tau_{1}>\sqrt{n} u \mid D=\infty\right] \\
& \leq P_{0}\left[\tau_{1}>\sqrt{n} u\right]+\frac{(n T+1)}{n u^{2}} E_{0}\left[\tau_{1}^{2}, \tau_{1}>\sqrt{n} u \mid D=\infty\right] \underset{n \rightarrow \infty}{\rightarrow} 0,
\end{aligned}
$$

using (4.3) and the $P_{0}$-a.s. finiteness of $\tau_{1}$ in the last step. Thus the Skorohoddistance (see Ethier-Kurtz [6], p. 117) of $B^{n}$ and $\frac{\Sigma_{k[\cdot n]}}{\sqrt{n}}$, tends to 0 in $P_{0^{-}}$ probability, as $n$ tends to infinity. Proving the convergence in law of the latter process to a Brownian motion with covariance matrix $A$, will thus imply a similar statement for $B^{n}$.

From Donsker's invariance principle, see for instance [6], p. 278, we know that

$$
\begin{aligned}
& \frac{\Sigma \cdot m}{\sqrt{m}} \text { converges in law to a } d \text {-dimensional Brownian motion with } \\
& \text { covariance matrix } E_{0}\left[\tau_{1} \mid D=\infty\right] A,
\end{aligned}
$$

provided $\Sigma_{s}, s \geq 0$, stands for the linear interpolation of $\Sigma_{m}, m \geq 0$. It then follows from (4.5) and (4.10) that the finite dimensional distribution of $\frac{\Sigma_{k \cdot n]}}{\sqrt{n}}$, converge to the finite dimensional distribution of a $d$-dimensional Brownian motion with covariance matrix $A$. Moreover the tightness of the laws of $\frac{\Sigma_{k[\cdot n]}}{\sqrt{n}}$ follows from (4.5), (4.10) and Corollary 7.4, p. 129 of [6]. This proves the asserted convergence in law of $B^{n}$.

There only remains to prove the nondegeneracy of $A$. This is where the assumption (4.2) will be convenient. Consider $w \in \mathbb{R}^{d}$ with ${ }^{t} w A w=0$, so that

$$
P_{0}\left[w \cdot\left(X_{\tau_{1}}-\tau_{1} v\right)=0 \mid D=\infty\right]=1 .
$$


The collection of $x \in \mathbb{Z}^{d}$ with $P_{0}\left[X_{S_{1}}=x, S_{1}<D\right]>0$, coincides thanks to the connectedness of $\left\{x \in \mathbb{Z}^{d}, 0 \leq x \cdot \ell<a\right\}$, with

$$
H=\partial\left\{z \in \mathbb{Z}^{d}, \ell \cdot z<a\right\} .
$$

We shall now see that:

$$
w \cdot v=0 \text { and } w \cdot x=0 \text {, for any } x \in H .
$$

Indeed consider $x \in H$, so that $P_{0}\left[X_{S_{1}}=x, S_{1}<D\right]>0$. Using a nearest neighbor loop of arbitrary length inside $\{z: 0 \leq \ell \cdot z<a\}$, starting and ending in 0 , we see that for all $n \geq 0$,

$$
P_{0}\left[X_{S_{1}}=x, n \leq S_{1}<D\right]>0 .
$$

Then as a result of the strong Markov property and the independence under $\mathbb{P}$, for $n \geq 0$, and $x \in H$ :

$$
\begin{aligned}
& P_{0}\left[X_{\tau_{1}}=x, n<\tau_{1}=S_{1}, D=\infty\right] \\
& \stackrel{(1.18)}{=} P_{0}\left[X_{S_{1}}=x, n<S_{1}<D, D \circ \theta_{S_{1}}=\infty\right] \\
& =\mathbb{E}\left[P_{0, \omega}\left[X_{S_{1}}=x, n<S_{1}<D\right] P_{x, \omega}[D=\infty]\right] \\
& =P_{0}\left[X_{S_{1}}=x, n<S_{1}<D\right] P_{0}[D=\infty]>0 .
\end{aligned}
$$

Thus in view of (4.11), for arbitrary $n \geq 0$ and $x \in H$ :

$$
n|w \cdot v| \leq|w \cdot x|,
$$

which implies $w \cdot v=0$. Coming back to (4.11), we now deduce (4.13). Then taking limits of points in $H$, we see that

$$
w \cdot y=0 \text {, for any } y \in \mathbb{R}^{d} \text {, orthogonal to } \ell \text {. }
$$

Since $v \cdot \ell>0,(4.13)$ and (4.17) imply that $w=0$. This proves that $A$ is non-degenerate.

As an immediate consequence of Theorem 3.5 we find:

Corollary 4.2. $(d \geq 2)$. Under (0.1) and (1.7),

$B^{n}$. converges in law to a Brownian motion with non-degenerate covariance matrix A, see (4.4).

Remark 4.3. In the one-dimensional case, it is known that (0.1), (1.7) does not necessarily ensure (4.18), we refer on this to Kesten-Kozlov-Spitzer [12]. 


\section{Slowdown estimates}

The main object of this section is to derive tail estimates on the probability that the walk moves slower in a direction $\ell \in S^{d-1}$, with respect to which Kalikow's condition (1.7) holds, than predicted by the strong law of large numbers (1.21). We shall mainly be concerned with estimates under the annealed probability $P_{0}$, but shall provide some comments at the end of the section on how annealed estimates can be used to derive quenched estimates. As mentioned in the introduction, there is an interplay between "traps" and "slowdown" of the process, and a very natural question of knowing whether or not "traps" govern tail estimates of slowdown probabilities. The present section will bring some elements of answer to this question; the key role of the variable $\tau_{1}$ will also be apparent. We shall also briefly discuss at the end of the section what happens when "slowdown" is replaced by "acceleration".

We begin with a useful lemma. We consider for $u \geq 0$, the random variables

$$
N_{u}=\inf \left\{k \geq 0, X_{\tau_{k}} \cdot \ell \geq u\right\}
$$

Lemma 5.1. $(d \geq 1)$. Assume (0.1) and (1.7). For $\rho>0$,

$$
\varlimsup_{u \rightarrow \infty} u^{-1} \log P_{0}\left[\left|\frac{N_{u}}{u}-\frac{1}{E_{0}\left[X_{\tau_{1}} \cdot \ell \mid D=\infty\right]}\right| \geq \rho\right]<0 .
$$

Moreover, for large $u$ and arbitrary $c>0$,

$$
P_{0}\left[T_{u}>c u\right] \geq P_{0}\left[\tau_{1}>c u\right]-e^{-\frac{c_{2}}{2} u} .
$$

Proof. We begin with the proof of (5.2). As an application of standard Cramer-type estimates, (1.19) and (1.32),

$\varlimsup_{k \rightarrow \infty} \frac{1}{k} \log P_{0}\left[\left|X_{\tau_{k}} \cdot \ell-k E_{0}\left[X_{\tau_{1}} \cdot \ell \mid D=\infty\right]\right| \geq \rho k\right]<0$, for $\rho>0$.

The claim (5.2) then follows by routine arguments.

We now turn to the proof of (5.3). Observe that for $u>0$ :

$$
P_{0} \text {-a.s., on }\left\{X_{\tau_{1}} \cdot \ell \leq u\right\}, \tau_{1}=T_{X_{\tau_{1}} \cdot \ell} \leq T_{u},
$$

so that for $u>0$ and $c>0$,

$$
P_{0}\left[\tau_{1}>c u\right] \leq P_{0}\left[T_{u}>c u\right]+P_{0}\left[X_{\tau_{1}} \cdot \ell>u\right] .
$$

The claim (5.3) now follows from Lemma 1.2. 
We first begin with the simpler situation of annealed slowdown estimates for non-nestling walks. The one-dimensional case is well known, cf. Grevenden Hollander [8], or Dembo-Peres-Zeitouni [4].

Theorem 5.2. $(d \geq 1)$. Assume (2.1), then for $c>(v \cdot \ell)^{-1}$, in the notation of $(0.5)$,

(5.4) $-\infty<\varliminf_{u \rightarrow \infty} u^{-1} \log P_{0}\left[T_{u}>c u\right] \leq \varlimsup_{u \rightarrow \infty} u^{-1} \log P_{0}\left[T_{u}>c u\right]<0$.

Proof. The lower bound is trivial. Let us prove the upper bound. Given $c>(v \cdot \ell)^{-1}$, let us pick $\tilde{c}$ such that

$$
\begin{aligned}
& \left(E_{0}\left[\tau_{1} \mid D=\infty\right] v \cdot \ell\right)^{-1}= \\
& \quad \frac{1}{E_{0}\left[X_{\tau_{1}} \cdot \ell \mid D=\infty\right]}<\widetilde{c}<E_{0}\left[\tau_{1} \mid D=\infty\right]^{-1} c .
\end{aligned}
$$

As a result of Lemma 5.1, we obtain:

$$
\varlimsup_{u \rightarrow \infty} u^{-1} \log P_{0}\left[N_{u} \geq \tilde{c} u\right]<0,
$$

and since $N_{u}<\tilde{c} u$, implies $\tau_{[\widetilde{c} u]+1} \geq T_{u}$, our claim will follow once we show:

$$
\varlimsup_{u \rightarrow \infty} u^{-1} \log P_{0}\left[\tau_{[\widetilde{c} u]+1}>c u\right]<0 .
$$

However since $E_{0}\left[\tau_{1} \mid D=\infty\right] \widetilde{c}<c$, the renewal property (1.19), together with the finiteness of some exponential moment of $\tau_{1}$ under $P_{0}$, cf. Theorem 2.1, implies (5.7) by standard Cramer-type estimates.

The next result will in particular apply to the situation of Theorem 3.5 and especially to the plain nestling situation.

Theorem 5.3. $(d \geq 1)$. Assume (0.1), (1.7) and that for some $\alpha>0$,

$$
\begin{gathered}
\varlimsup_{u \rightarrow \infty}(\log u)^{-\alpha} \log P_{0}\left[\tau_{1}>u\right]<0, \text { then } \\
\varlimsup_{u \rightarrow \infty}(\log u)^{-\alpha} \log P_{0}\left[T_{u}>c u\right]<0, \text { for } c>(v \cdot \ell)^{-1} .
\end{gathered}
$$

Proof. Let us first mention that when $d=1$, only the statement with $\alpha=1$ has some relevance, cf. Remark 5.6 1) below, and when $d \geq 2$, one can in fact assume that (5.8) holds with $\alpha>1$, thanks to Theorem 3.5. Keeping these comments in mind, let us begin with the proof of (5.9). If we choose $\widetilde{c}$, as in (5.5), a similar argument shows that (5.9) follows from: 


$$
\varlimsup_{u \rightarrow \infty}(\log u)^{-\alpha} \log P_{0}\left[\tau_{[\widetilde{c} u]+1}>c u\right]<0 .
$$

Furthermore, in view of (1.19), (5.8) and the inequality

$$
E_{0}\left[\tau_{1} \mid D=\infty\right] \tilde{c}<c,
$$

it suffices to show that for $\delta>0$,

$$
\varlimsup_{n \rightarrow \infty}(\log n)^{-\alpha} \log P\left[\widetilde{\tau}_{1}+\cdots+\widetilde{\tau}_{n} \geq n(\tilde{m}+\delta)\right]<0,
$$

when $\widetilde{\tau}_{i}, i \geq 1$, under $P$ are independent, all distributed like $\tau_{1}$ under $P_{0}[\cdot \mid D=\infty]$, and

$$
\widetilde{m}=E_{0}\left[\tau_{1} \mid D=\infty\right] .
$$

As a result of Chebyshev inequality and independence, for $n>1$,

$$
\begin{aligned}
& P\left[\widetilde{\tau}_{1}+\cdots+\widetilde{\tau}_{n} \geq n(\tilde{m}+\delta)\right] \leq n P_{0}\left[\widetilde{\tau}_{1}>n(\log n)^{-\alpha-1}\right]+ \\
& P\left[\widetilde{\tau}_{1}+\cdots+\widetilde{\tau}_{n} \geq n(\widetilde{m}+\delta), \sup _{1 \leq i \leq n} \widetilde{\tau}_{i} \leq n(\log n)^{-\alpha-1}\right],
\end{aligned}
$$

so that with the help of (5.8), for a suitable $\gamma>0$, and large $n$,

$$
\begin{aligned}
\leq n \exp \left\{-\gamma\left(\log \frac{n}{(\log n)^{\alpha+1}}\right)^{\alpha}\right\}+ & \exp \left\{-(\log n)^{\alpha}(\widetilde{m}+\delta)\right\} \\
& E\left[\exp \left\{\frac{(\log n)^{\alpha}}{n} \widetilde{\tau}_{1}\right\}, \widetilde{\tau}_{1} \leq \frac{n}{(\log n)^{\alpha+1}}\right]^{n} .
\end{aligned}
$$

However, for large $n$,

$$
\begin{aligned}
& E {\left[\exp \left\{\frac{(\log n)^{\alpha}}{n} \widetilde{\tau}_{1}\right\}, \widetilde{\tau}_{1} \leq \frac{n}{(\log n)^{\alpha+1}}\right] } \\
&(5.14) \quad \leq 1+\int_{0}^{\frac{n}{\log n)} \alpha+1} \frac{(\log n)^{\alpha}}{n} \exp \left\{\frac{(\log n)^{\alpha}}{n} u\right\} P\left[\widetilde{\tau}_{1}>u\right] d u \\
& \quad \leq 1+e^{(\log n)^{-1}} \frac{(\log n)^{\alpha}}{n} \int_{0}^{\infty} P\left[\widetilde{\tau}_{1}>u\right] d u \leq 1+\frac{(\log n)^{\alpha}}{n}\left(\widetilde{m}+\frac{\delta}{2}\right) .
\end{aligned}
$$

Coming back to (5.13), we see that for large $n$ :

$$
\begin{aligned}
P\left[\widetilde{\tau}_{1}+\cdots+\widetilde{\tau}_{n} \geq n(\widetilde{m}+\delta)\right] \leq \\
\quad \exp \left\{-\frac{\gamma}{2}(\log n)^{\alpha}\right\}+\exp \left\{-(\log n)^{\alpha}\left(\widetilde{m}+\delta-\widetilde{m}-\frac{\delta}{2}\right)\right\} .
\end{aligned}
$$

The claim (5.11) follows. 
Collecting theorems 3.5 and 5.3, we obtain

Corollary 5.4. $(d \geq 2)$. Under (0.1) and (1.7), for $\alpha<1+\frac{d-1}{3 d}$, and $c>(v \cdot \ell)^{-1}$,

$$
\varlimsup_{u \rightarrow \infty}(\log u)^{-\alpha} \log P_{0}\left[T_{u}>c u\right]<0 .
$$

In the case of plain nestling walks, we also have the following lower bound:

Theorem 5.5. $(d \geq 1)$. When (0.1), (1.7) hold and the walk is plain nestling,

$$
\varliminf_{u \rightarrow \infty}(\log u)^{-d} \log P_{0}\left[T_{u}>c u\right]>-\infty, \text { when } c>0 .
$$

Proof. We simply apply (5.3) together with Theorem 2.7.

Remark 5.6. 1) In the one-dimensional plain nestling situation, when (1.7) holds, it is shown in Theorem 1.1 of Dembo-Peres-Zeitouni [4] that

$$
\lim _{u \rightarrow \infty}(\log u)^{-1} \log P_{0}\left[T_{u}>c u\right]=1-s<0, \text { for } c>(v \cdot \ell)^{-1},
$$

where $s$ is the unique zero bigger than 1 of the convex function

$$
F(t)=\log \mathbb{E}\left[\left(\frac{\omega(0,-\ell)}{\omega(0, \ell)}\right)^{t}\right] .
$$

2) It is natural to wonder whether in Corollary 5.4, (5.15) also holds with $\alpha=d$.

3) It is not hard to modify the arguments we used, and show that under the respective assumptions of Theorem 5.3 or Corollary 5.4, for $0<v_{1}<v \cdot \ell$,

$$
\varlimsup_{n}(\log n)^{-\alpha} \log P_{0}\left[\frac{X_{n} \cdot \ell}{n}<v_{1}\right]<0,
$$

with $\alpha$ respectively as in Theorem 5.3 or Corollary 5.4. Indeed observe that for $0<v_{1}<v_{2}<v \cdot \ell$,

$$
\begin{aligned}
& P_{0}\left[X_{n} \cdot \ell<v_{1} n\right] \leq P_{0}\left[T_{v_{2} n}>n\right]+ \\
& P_{0}\left[T_{v_{2} n} \leq n, \widetilde{T}_{v_{1} n} \circ \theta_{T_{v_{2} n}}<\infty\right] .
\end{aligned}
$$

Using essentially the arguments below (1.41), see (1.43) and (1.44), we see that the second term of the right hand side of (5.20) has an exponential decay. The claim (5.19) now follows from the application of Theorem 5.3 (respectively Corollary 5.4) to the first term of the right member of (5.20). 
4) It is also not hard to modify the argument in the proof of Theorem 5.5, to show that under the same assumptions, when $0<v_{1}<v_{2}<v \cdot \ell$,

$$
\varliminf_{n \rightarrow \infty}(\log n)^{-d} \log P_{0}\left[\frac{X_{n} \cdot \ell}{n} \in\left(v_{1}, v_{2}\right)\right]>-\infty .
$$

Indeed keeping the notations of Theorem 5.5, one can find when $r \rightarrow \infty$, $x_{0}$ remaining within bounded distance from $B_{r}$, with $x_{0} \cdot \ell>r$, and using a similar argument as in (2.55), and (0.1), show that for any $0<c_{1}<c_{2}$, for a suitable $r=0(\log n)$,

$$
\varliminf_{n \rightarrow \infty}(\log n)^{-d} \log P_{0}\left[T_{x_{0} \cdot \ell}=H_{x_{0}} \in\left(c_{1} n, c_{2} n\right)\right]>-\infty .
$$

Observe also that in view of the strong law of large numbers, and the fact that $\left|X_{k+1}-X_{k}\right|=1, P_{0}$-a.s.,

$$
\sup _{0 \leq k \leq n}\left|\frac{X_{k}}{n}-\frac{k}{n} v\right| \rightarrow 0, P_{0}[\cdot \mid D=\infty] \text {-a.s., as } n \rightarrow \infty \text {. }
$$

Thus choosing $c_{1}<c_{2}$, close to $c_{0}$ such that $(v \cdot \ell)\left(1-c_{0}\right)=\frac{v_{1}+v_{2}}{2}$, and $\rho>0$, small enough, for large $n$ :

$$
\begin{aligned}
P_{0}\left[X_{n} \cdot \ell \in\right. & \left.n\left(v_{1}, v_{2}\right)\right] \geq P_{0}\left[T_{x_{0} \cdot \ell}=H_{x_{0}} \in n\left(c_{1}, c_{2}\right),\right. \\
& \left.D \circ \theta_{H_{x_{0}}}=\infty, \sup _{0 \leq k \leq n}\left|\left(X_{k+H_{x_{0}}}-x_{0}\right)-k v\right| \leq \rho n\right] .
\end{aligned}
$$

Using the strong Markov property, independence, (5.22) and (5.23), we see that

$$
\begin{array}{ll}
\varliminf_{n \rightarrow \infty} & (\log n)^{-d} \log P_{0}\left[X_{n} \cdot \ell \in n\left(v_{1}, v_{2}\right)\right] \geq \\
\underset{n \rightarrow \infty}{\lim }(\log n)^{-d} \log P_{0}\left[T_{x_{0} \cdot \ell}=H_{x_{0}} \in n\left(c_{1}, c_{2}\right)\right]+ & \\
\varliminf_{n \rightarrow \infty}(\log n)^{-d} \log P_{0}\left[D=\infty, \sup _{0 \leq k \leq n}\left|\frac{X_{k}}{n}-\frac{k}{n} v\right| \leq \rho\right]>-\infty .
\end{array}
$$

This proves (5.21).

The next result will in particular enable to apply estimates like (2.16) to the derivation of upper bounds on the probability of slowdowns, in the marginal nestling situation.

Theorem 5.7. $(d \geq 1)$. Assume (0.1), (1.7), and for some $\alpha \geq 1$ :

$$
\begin{gathered}
\varlimsup_{u \rightarrow \infty} u^{-\frac{\alpha}{\alpha+2}} \log P_{0}\left[\tau_{1}>u\right]<0, \text { then } \\
\varlimsup_{u \rightarrow \infty} u^{-\frac{\alpha}{\alpha+2}} \log P_{0}\left[T_{u}>c u\right]<0, \text { for } c>(v \cdot \ell)^{-1} .
\end{gathered}
$$


Proof. By the same reasoning as in the proof of Theorem 5.3, it suffices to show that for $\delta>0$,

$$
\varlimsup_{n \rightarrow \infty} n^{-\frac{\alpha}{\alpha+2}} \log P\left[\widetilde{\tau}_{1}+\cdots+\widetilde{\tau}_{n} \geq n(\widetilde{m}+\delta)\right]<0 .
$$

where $\widetilde{\tau}_{i}$ are independent under $P$ and distributed like $\tau_{1}$ under $P_{0}[\cdot \mid D=\infty]$. Observe that (5.25) implies in view of the proof of (5.11), that for any $\beta>1$ :

$$
\varlimsup_{n \rightarrow \infty}(\log n)^{-\beta} \log P\left[\widetilde{\tau}_{1}+\cdots+\widetilde{\tau}_{n} \geq n(\widetilde{m}+\delta)\right]<0 .
$$

For large $n$, we divide $\{1, \ldots, n\}$ into $M \leq\left[\frac{n}{\frac{1}{\alpha+2}}\right]+1$ consecutive blocks, $B_{1}, \ldots, B_{M}$ of $\left[n^{\frac{1}{\alpha+2}}\right]$ integers except may be for the "last one". Pick now some $\beta>1$. By a very rough counting argument, the number of subsets of size $\left[\frac{n \frac{\alpha}{\alpha+2}}{(\log n)^{\beta}}\right] \stackrel{\text { def }}{=} a_{n}$ in $\{1, \ldots, M\}$ grows like $\exp \left\{o\left(n^{\frac{\alpha}{\alpha+2}}\right)\right\}$. Moreover, if

$$
A_{n} \stackrel{\text { def }}{=}\left\{\sum_{j=1}^{M} 1\left\{\sum_{i \in B_{j}} \tilde{\tau}_{i}>\left(\tilde{m}+\frac{\delta}{2}\right)\left[n^{\frac{1}{\alpha+2}}\right]\right\} \geq \frac{n^{\frac{\alpha}{\alpha+2}}}{(\log n)^{\beta}}\right\},
$$

on $A_{n}$ one can find a subset of size $a_{n}$ in $\{1, \ldots, M\}$, such that for each block with label in this subset, the corresponding sum of $\widetilde{\tau}_{i}$ exceeds $\left(\widetilde{m}+\frac{\delta}{2}\right)\left[n^{\frac{1}{\alpha+2}}\right]$. As a result,

$$
P\left(A_{n}\right) \leq \exp \left\{o\left(n^{\frac{\alpha}{\alpha+2}}\right)\right\} P\left[\widetilde{\tau}_{1}+\cdots+\widetilde{\tau}_{\left[n^{\frac{1}{\alpha+2}}\right]}>\left(\widetilde{m}+\frac{\delta}{2}\right)\left[n^{\frac{1}{\alpha+2}}\right]\right]^{a_{n}},
$$

the latter quantity in view of (5.28), for a suitable $\gamma>0$, is smaller for large $n$ than:

$$
\exp \left\{o\left(n^{\frac{\alpha}{\alpha+2}}\right)-\gamma\left(\log \left[n^{\frac{1}{\alpha+2}}\right]\right)^{\beta} a_{n}\right\} \leq \exp \left\{-\gamma^{\prime} n^{\frac{\alpha}{\alpha+2}}\right\} \text {, with } \gamma^{\prime}>0 .
$$

The claim (5.27) will therefore follow once we prove:

$$
\varlimsup_{n \rightarrow \infty} n^{-\frac{\alpha}{\alpha+2}} \log P\left[\widetilde{\tau}_{1}+\cdots+\widetilde{\tau}_{n}>n(\tilde{m}+\delta), A_{n}^{c}\right]<0 .
$$

Denote by $\mathcal{J}$ the random set of indices $j$ in $\{1, \ldots, M\}$ of blocks $B_{j}$ such that $\sum_{i \in B_{j}} \widetilde{\tau}_{i}>\left(\widetilde{m}+\frac{\delta}{2}\right)\left[n^{\frac{1}{\alpha+2}}\right]$. Observe that for large $n, \widetilde{\tau}_{1}+\cdots+\widetilde{\tau}_{n}>(\widetilde{m}+\delta) n$ implies that $\sum_{j \in \mathcal{J}} \sum_{i \in B_{j}} \widetilde{\tau}_{i}>\frac{\delta}{4} n$. Moreover, by a similar rough counting argument as above, the number of subsets of $\{1, \ldots, M\}$ with cardinality smaller than $n^{\frac{\alpha}{\alpha+2}}(\log n)^{-\beta}$ grows like $\exp \left\{o\left(n^{\frac{\alpha}{\alpha+2}}\right)\right\}$. As a result for large $n$, with $b_{n} \stackrel{\text { def }}{=} a_{n}\left[n^{\frac{1}{\alpha+2}}\right] \leq n^{\frac{\alpha+1}{\alpha+2}}(\log n)^{-\beta}$,

$$
\begin{aligned}
P\left[\widetilde{\tau}_{1}+\cdots+\widetilde{\tau}_{n}>\right. & \left.n(\widetilde{m}+\delta), A_{n}^{c}\right] \leq \\
& \quad \exp \left\{o\left(n^{\frac{\alpha}{\alpha+2}}\right)\right\} P\left[\widetilde{\tau}_{1}+\cdots+\widetilde{\tau}_{b_{n}}>\frac{\delta}{4} n\right],
\end{aligned}
$$


and for $\lambda>0$,

$$
\begin{aligned}
& \leq \exp \left\{o\left(n^{\frac{\alpha}{\alpha+2}}\right)\right\}\left(n^{\frac{\alpha+1}{\alpha+2}}(\log n)^{-\beta} P\left[\tilde{\tau}_{1}>\frac{\delta}{4} n\right]\right. \\
& \left.+\exp \left\{-\lambda \frac{\delta}{4} n^{\frac{\alpha}{\alpha+2}}\right\} \mathbb{E}\left[\exp \left\{\lambda n^{-\frac{2}{\alpha+2}} \widetilde{\tau}_{1}\right\}, \widetilde{\tau}_{1}<\frac{\delta}{4} n\right]^{b_{n}}\right) .
\end{aligned}
$$

Observe also that in view of (5.25), for suitable $\gamma_{1}, \gamma_{2}>0$,

$$
P\left[\widetilde{\tau}_{1}>u\right] \leq \gamma_{1} \exp \left\{-\gamma_{2} u^{\frac{\alpha}{\alpha+2}}\right\}, \text { for } u \geq 0,
$$

and as a result, for large $n$ :

$$
\begin{aligned}
E\left[\exp \left\{\lambda n^{-\frac{2}{\alpha+2}} \widetilde{\tau}_{1}\right\}, \widetilde{\tau}_{1}<\frac{\delta}{4} n\right] \\
\quad \leq 1+\frac{\lambda}{n^{\frac{2}{\alpha+2}}} \int_{0}^{\frac{\delta}{4} n} \gamma_{1} \exp \left\{\frac{\lambda}{\frac{2}{n^{\alpha+2}}} u-\gamma_{2} u^{\frac{\alpha}{\alpha+2}}\right\} d u \\
\leq 1+\gamma_{3} n^{-\frac{2}{\alpha+2}}
\end{aligned}
$$

provided we choose $\lambda>0$, small enough. Inserting this estimate in the rightmost handside of (5.32), we obtain (5.31). This finishes the proof of the theorem.

As an immediate application of the theorem we obtain a result which improves our previous results of [24] on walks which are neutral or biased to the right.

Theorem 5.8. $(d \geq 1)$. In the case of a walk which is neutral or biased to the right (i.e. under (2.37), (2.38)),

$$
\begin{aligned}
-\infty<\varliminf_{u \rightarrow \infty} u^{-\frac{d}{d+2}} \log P_{0}\left[T_{u}>c u\right] \leq \\
\varlimsup_{u \rightarrow \infty} u^{-\frac{d}{d+2}} \log P_{0}\left[T_{u}>c u\right]<0, \text { for } c>\left(v \cdot \ell_{1}\right)^{-1} .
\end{aligned}
$$

Proof. The upper bound is a consequence of Theorem 5.7 and Theorem 2.5, (one can also use Proposition 2.3 in place of Theorem 2.5 when $d=1$ ). As for the lower bound, it is proven with the help of (2.39) and (5.3).

Let us close this section with a few remarks. In particular we discuss what happens when "acceleration" replaces "slowdown", and "quenched" probabilities are considered instead of "annealed" probabilities. We also characterize, when (0.1) and (1.7) hold, the location of the null set of the rate function entering the quenched large deviation proven by Zerner in the nestling case, cf. [26].

Remark 5.9. 1) By similar arguments as in the proof of (5.19) and (5.21), we see that under the assumptions of Theorem 5.8, for $0<v_{1}<v_{2}<v \cdot \ell$, 


$$
-\infty<\varlimsup_{n \rightarrow \infty} n^{-\frac{d}{d+2}} \log P_{0}\left[\frac{X_{n} \cdot \ell}{n} \in\left(v_{1}, v_{2}\right)\right]<0 .
$$

2) Tail estimates on the annealed probability of acceleration of the walk in the direction $\ell$, when $(0.1),(1.7)$ hold, do not display the same variety as in the case of slowdowns. Indeed Lemma 5.1, together with Cramer-type estimates and a simple truncation argument shows that one always has:

$$
\varlimsup_{u \rightarrow \infty} u^{-1} \log P_{0}\left[T_{u}<c u\right]<0, \text { for } c<(v \cdot \ell)^{-1} .
$$

This immediately implies that

$$
\varlimsup_{n \rightarrow \infty} \frac{1}{n} \log P_{0}\left[\frac{X_{n} \cdot \ell}{n}>v_{1}\right]<0, \text { for } v_{1}>v \cdot \ell .
$$

3) In the nestling case, if for instance (0.1) holds, M. Zerner [26], cf. Theorem B, has shown that:

$$
\begin{aligned}
& \mathbb{P} \text {-a.s., } \frac{X_{n}}{n} \text { satisfies a large deviation principle under } P_{0, \omega} \\
& \text { at rate } n \text { with a deterministic convex continuous rate } \\
& \text { function } I(\cdot): \mathbb{R}^{d} \rightarrow[0, \infty], \text { which can be expressed } \\
& \text { as } I(\cdot)=\sup _{\lambda \geq 0}\left(\alpha_{\lambda}(\cdot)-\lambda\right),
\end{aligned}
$$

$\left(\alpha_{\lambda}(\cdot)\right.$ are the so-called Lyapunov coefficients).

The location of the null set of $I(\cdot)$ is so far poorly understood. Let us however mention that

Proposition 5.10. Assume that (0.1), (1.7) hold and the walk is nestling, then

$$
\{I(\cdot)=0\}=\{\lambda v, 0 \leq \lambda \leq 1\} .
$$

Proof. Indeed $\alpha_{0} \leq I$, and the inclusion of $\{I(\cdot)=0\}$ in $\mathbb{R}_{+} v$ follows from (1.70). Moreover from (5.38) and a Borel-Cantelli argument, we see that:

$$
\mathbb{P} \text {-a.s., } \varlimsup_{n \rightarrow \infty} \frac{1}{n} \log P_{0, \omega}\left[\frac{X_{n} \cdot \ell}{n}>v_{1}\right]<0 \text {, for } v_{1}>v \cdot \ell \text {. }
$$

This and the above mentioned large deviation principle shows that inf $\{I(x)$; $\left.\ell \cdot x>v_{1}\right\}>0$, for $v_{1}>\ell \cdot v$. Thus $\{I(\cdot)=0\} \subseteq\{\lambda v, 0 \leq \lambda \leq 1\}$.

It is also a simple matter, using the fact that (cf. Proposition 8 of [26]):

$$
\mathbb{P} \text {-a.s., } \varlimsup_{n} \frac{1}{n} \log P_{0, \omega}\left[X_{n}=0\right]=0,
$$

to deduce the inclusion:

$$
\{u v, 0 \leq u \leq 1\} \subseteq\{I(\cdot)=0\} .
$$

This concludes the proof of (5.40). 
4) The results of this section have direct applications to the derivation of quenched probabilities of slowdown, essentially by adapting the proof used in Sect. V of [24], (see also Gantert-Zeitouni [7] for the one-dimensional case). To keep a reasonable length to this already long article, we refrain from developping here in details all these quenched estimates. Let us however mention, that:

- in the non-nestling situation, i.e. under (0.1) and (2.1), on a set of full $\mathbb{P}$-measure, for $c>(v \cdot \ell)^{-1}$,

$$
\begin{aligned}
-\infty<\varliminf_{u \rightarrow \infty} u^{-1} \log P_{0, \omega}\left[T_{u}\right. & >c u] \\
& \leq \varlimsup_{u \rightarrow \infty} u^{-1} \log P_{0, \omega}\left[T_{u}>c u\right]<0,
\end{aligned}
$$

- for walks which are neutral or biased to the right, i.e. under (0.1), (2.37), (2.38), on a set of full $\mathbb{P}$-measure, for $c>\left(v \cdot e_{1}\right)^{-1}$

$$
\begin{aligned}
-\infty<\varliminf_{u \rightarrow \infty} \frac{\log u)^{\frac{2}{d}}}{u} & \log P_{0, \omega}\left[T_{u}>c u\right] \\
& \leq \varlimsup_{u \rightarrow \infty} \frac{(\log u)^{\frac{2}{d}}}{u} \log P_{0, \omega}\left[T_{u}>c u\right]<0,
\end{aligned}
$$

- for plain nestling walks satisfying (0.1), (1.7), for a suitable $A>0$, on a set of full $\mathbb{P}$-measure, for $c>0$

$$
\varliminf_{u \rightarrow \infty} u^{-1} \exp \left\{A(\log u)^{\frac{1}{d}}\right\} \log P_{0, \omega}\left[T_{u}>c u\right]>-\infty,
$$

and under the assumptions of Theorem 3.5, for each $c>(v \cdot \ell)^{-1}$, and $\alpha \in\left(1,1+\frac{d-1}{3 d}\right)$, there exists $B(c, \alpha)>0$, such that on a set of full $\mathbb{P}$-measure:

$$
\varlimsup_{u \rightarrow \infty} u^{-1} \exp \left\{B(\log u)^{\frac{1}{\alpha}}\right\} \log P_{0, \omega}\left[T_{u}>c u\right]<0 .
$$

Let us give some comments on the proofs. The upper bound in (5.42) follows from (5.2) by a Borel-Cantelli argument, and the lower bound is immediate. As for the proofs of (5.43)-(5.45), they are merely variations on the arguments of Sect. V of [24], explaining how to infer quenched statements from annealed statements. The lower bounds follow by BorelCantelli considerations showing for typical $\omega$ the existence of "traps" (i.e. neutral pockets or in the sense of (2.51)) with logarithmic size, within distance $o(u)$ from the origin. As for the upper bounds, one picks $c^{\prime} \in\left(\frac{1}{v \cdot \ell}, c\right)$, and slices the space along the direction $\ell$ into "slabs" of size $r_{u}$ such that:

$$
\varlimsup_{u \rightarrow \infty} \frac{\log P_{0}\left[T_{r_{u}}>c^{\prime} r_{u}\right]}{\log u}<-\left(2+d+4 c^{\prime}\right),
$$


which leads to a choice $r_{u}=$ const $(\log u)^{1+\frac{2}{a}}$, when proving (5.43), $r_{u}=$ $\exp \left\{\right.$ const $\left.(\log u)^{\frac{1}{\alpha}}\right\}$, when proving (5.45). If one introduces

$$
\begin{aligned}
& \widetilde{S}_{1}=\inf \left\{n \geq 0,\left|X_{n} \cdot \ell-X_{0} \cdot \ell\right| \geq r_{u}\right\} \\
& \widetilde{T}=\inf \left\{n \geq 0, \quad X_{n} \cdot \ell-X_{0} \cdot \ell \geq r_{u}\right\},
\end{aligned}
$$

then Borel-Cantelli considerations, with the help of (1.44), (1.47) and (5.46) show that

$$
\begin{aligned}
& \mathbb{P} \text {-a.s., for large integer } u, \text { for } x \in C_{4 u}, \\
& P_{x, \omega}\left[\widetilde{T}>c^{\prime} r_{u}\right] \leq u^{-4 c^{\prime}} \\
& P_{x, \omega}[\widetilde{S} \neq \widetilde{T}] \leq u^{-4 c^{\prime}} \\
& P_{0, \omega}\left[T_{C_{4 u}}<T_{2 u}\right] \leq \varphi(u)
\end{aligned}
$$

with $\varphi(u)=\exp \left\{-\right.$ const $\left.\frac{u}{(\log u)^{\frac{2}{d}}}\right\}$, when proving (5.43), and $\varphi(u)=$ $\exp \left\{-u e^{-\operatorname{const}(\log u)^{\frac{1}{\alpha}}}\right\}$, when proving (5.45). This essentially corresponds to the estimates of Lemma 5.1 of [24]. The proof then proceeds as in [24], with minor modifications.

Acknowledgement. We wish to thank M. Zerner and the referees for their suggestions and their careful reading of the original draft of this article.

\section{References}

1. Alon, N., Spencer, J., Erdös, P.: The probabilistic method. New York: John Wiley \& Sons 1992

2. Bricmont, J., Kupiainen, A.: Random walks in asymmetric random environments. Commun. Math. Phys. 142(2), 345-420 (1991)

3. Chow, Y.S., Teicher, H.: Probability theory. Second edition. New York: Springer 1988

4. Dembo, A., Peres, Y., Zeitouni, O: Tail estimates for one-dimensional random walk in random environment. Commun. Math. Phys. 181, 667-683 (1996)

5. Durrett, R.: Probability: Theory and Examples. Wadsworth and Brooks/Cole, Pacific Grove 1991

6. Ethier, S.M., Kurtz, T.G., Markov processes. New York: John Wiley \& Sons 1986

7. Gantert, N., Zeitouni, O.: Quenched sub-exponential tail estimates for one-dimensional random walk in random environment. Commun. Math. Phys. 194, 166-190 (1998)

8. Greven, A., den Hollander, F.: Large deviations for a random walk in random environment. Ann. Probab. 22, 1381-1428 (1994)

9. Kalikow, S.A.: Generalized random walk in a random environment. Ann. Probab. 9, 753-768 (1981)

10. Khas'minskii, R.Z.: On positive solutions of the equation $A u+V u=0$. Theoret. Probab. Appl. 3, 309-318 (1959)

11. Kesten, H.: A renewal theorem for random walk in a random environment. Proc. Symposia Pure Math. 31, 67-77 (1977)

12. Kesten, H., Kozlov, M.V., Spitzer, F.: A limit law for random walk in a random environment. Composito Mathematica 30(2), 145-168 (1975) 
13. Kipnis, C., Varadhan, S.R.S.: A central limit theorem for additive functionals of reversible Markov processes and applications to simple exclusions. Commun. Math. Phys. 104, 1-19 (1986)

14. Kozlov, S.M.: The method of averaging and walks in inhomogeneous environments. Russian Math. Surveys 40(2), 73-145 (1985)

15. Lawler, G.F.: Weak convergence of a random walk in a random environment. Commun. Math. Phys. 87, 81-87 (1982)

16. Martinelli, F.: Lectures on spin dynamics for discrete spin models. In: Ecole d'été de St Flour, Lecture Notes in Math. New York: Springer 1998

17. Molchanov, S.A.: Lectures on random media. Ecole d'été de Probabilités de St. Flour XXII-1992, Lecture Notes in Math. Vol. 1581, pp. 242-411. Heidelberg: Springer 1994

18. Olla, S.: Homogenization of diffusion processes in random fields. Ecole Doctorale, Ecole Polytechnique, Palaiseau (1994)

19. Papanicolaou, G., Varadhan, S.R.S.: Boundary value problems with rapidly oscillating random coefficients. In: Random Fields. Fritz, J., Szasz, D. (eds.), North-Holland: Janyos Bolyai series 1981

20. Pisztora, A., Povel, T.: Large deviation principle for random walk in a quenched random environment in the low speed regime. Ann Probab. 27(3), 1389-1413 (1999)

21. Pisztora, A., Povel, T., Zeitouni, O.: Precise large deviation estimates for onedimensional random walk in random environment. Probab. Theory Relat. Fields 113, 191-219 (1999)

22. Solomon, F.: Random walks in a random environment. Ann. Probab. 3, 1-31 (1975)

23. Sznitman, A.-S.: Brownian motion, obstacles and random media. Berlin: Springer 1998

24. Sznitman, A.-S.: Slowdown and neutral pockets for a random walk in random environment. Probab. Theory Relat. Fields 115, 287-323 (1999)

25. Sznitman, A.-S., Zerner, M.P.W.: A law of large numbers for random walks in random environment. Ann. Probab. 27(4), 1851-1869 (1999)

26. Zerner, M.P.W.: Lyapunov exponents and quenched large deviation for multidimensional random walk in random environment. Ann. Probab. 26, 1446-1476 (1998) 